Review

\title{
Exploring Light Sterile Neutrinos at Long Baseline Experiments: A Review
}

\author{
Antonio Palazzo 1,2 \\ 1 Dipartimento Interateneo di Fisica "Michelangelo Merlin”, University of Bari, Via Amendola 173, \\ I-70126 Bari, Italy; palazzo@ba.infn.it \\ 2 Istituto Nazionale di Fisica Nucleare (INFN), Sezione di Bari, Via E. Orabona 4, I-70126 Bari, Italy
}

Received: 10 January 2020; Accepted: 28 February 2020; Published: 7 March 2020

check for updates

\begin{abstract}
Several anomalies observed in short-baseline neutrino experiments suggest the existence of new light sterile neutrino species. In this review, we describe the potential role of long-baseline experiments in the searches of sterile neutrino properties and, in particular, the new $\mathrm{CP}$-violation phases that appear in the enlarged $3+1$ scheme. We also assess the impact of light sterile states on the discovery potential of long-baseline experiments of important targets such as the standard 3-flavor $\mathrm{CP}$ violation, the neutrino mass hierarchy, and the octant of $\theta_{23}$.
\end{abstract}

Keywords: neutrino oscillations; long-baseline; sterile neutrino; DUNE; T2HK; ESSvSB

\section{Introduction}

More than twenty years ago, groundbreaking observations of neutrinos produced in natural sources (sun and Earth atmosphere) provided the first evidence of neutrino oscillations and established the massive nature of neutrinos [1-5]. Subsequently, the neutrino properties have been clarified by experiments using man-made sources of neutrinos (nuclear reactors and accelerators). The two simple 2-flavor descriptions adopted in the beginning to describe disjointly the solar and the atmospheric neutrino problems have been gradually recognized as two pieces of a single picture, which is presently considered as the standard framework of neutrino oscillations. We recall that in the 3-flavor scheme there are three mass eigenstates $v_{i}$ with masses $m_{i}(i=1,2,3)$ [and therefore two independent mass-squared splittings $\left.\left(\Delta m_{31}^{2}, \Delta m_{21}^{2}\right)\right]$, three mixing angles $\theta_{12}, \theta_{23}, \theta_{13}$, and one CP-phase $\delta$. The neutrino mass hierarchy (NMH) is defined to be normal (NH) if $m_{3}>m_{1,2}$ or inverted (IH) if $m_{3}<m_{1,2}$.

Despite its huge success, the standard 3-flavor scheme does not need to constitute an exact description. As a matter of fact, several anomalies have been found in short baseline experiments (SBL), which cannot be explained in the 3-flavor scenario (for reviews on this subject, see [6-15]). The indications come from the accelerator experiments LSND [16] and MiniBooNE [17], and from the so-called reactor [18] and Gallium [19,20] anomalies. Constraints on light sterile neutrinos have been derived also by the long-baseline (LBL) experiments MINOS and MINOS+ [21,22], $\mathrm{NO} v \mathrm{~A}$ [23], and T2K [24]; by the reactor experiments Daya Bay [25], ${ }^{1}$ DANSS [27], NEOS [28], Neutrino-4 [29], PROSPECT [30], and STEREO [31]; by the atmospheric neutrino data collected in Super-Kamiokande [32], IceCube [33,34], and ANTARES [35]; and by solar neutrinos [36-38].

The two standard mass-squared splittings $\Delta m_{21}^{2} \equiv m_{2}^{2}-m_{1}^{2}$ and $\Delta m_{31}^{2} \equiv m_{3}^{2}-m_{1}^{2}$ are too small to give rise to detectable effects in SBL setups. Therefore, one new much bigger mass-squared difference

1 We also mention the work [26], where the combination of MINOS, Daya-Bay, and Bugey-3 was considered. 
$\mathrm{O}\left(\mathrm{eV}^{2}\right)$ must be invoked to explain the SBL anomalies. The new hypothetical mass eigenstate must be supposed to be sterile, i.e., a singlet of the standard model gauge group. Several new and more sensitive SBL experiments are underway to put under test such an intriguing hypothesis (see the review in [39]), which, if confirmed would provide a concrete evidence of physics beyond the standard model. In the minimal extension, the so-called $3+1$ scheme, only one sterile species is introduced. In this scenario, one assumes the existence of one mass eigenstate $v_{4}$ weakly mixed with the active neutrino flavors $\left(v_{e}, v_{\mu}, v_{\tau}\right)$ and separated from the standard mass eigenstates $\left(v_{1}, v_{2}, v_{3}\right)$ by a $O\left(\mathrm{eV}^{2}\right)$ difference. In the $3+1$ framework, there are six mixing angles and three (Dirac) CP-violating phases. Therefore, in the eventuality of a discovery of a light sterile species, we would face the daunting task of nailing down six new properties (three mixing angles, two CP-phases, and the mass-squared splitting $\Delta m_{41}^{2}$ ). Several global analyses performed in the $3+1$ scheme [12-15] show that the fit noticeably improves with respect to the standard 3-flavor case. However, all such analyses evidence an internal tension between different classes of experiments. More specifically, a tension among the joint disappearance data $\left(v_{e} \rightarrow v_{e}\right.$ and $\left.v_{\mu} \rightarrow v_{\mu}\right)$ and the appearance data $\left(v_{\mu} \rightarrow v_{e}\right)$ is present and has become stronger in the latest analyses. This has led to increasingly lower values for the best fit of $\theta_{14}$ and especially $\theta_{24}$, which currently are close [13] to $\theta_{14}=8^{\circ}$ and $\theta_{24}=7^{\circ}$, and are slightly smaller than the values that we have taken as a benchmark in this review $\left(\theta_{14}=\theta_{24}=9^{\circ}\right)$.

The $3+1$ scenario naturally implies sizeable effects at short distances, where the oscillation factor $\Delta_{41} \equiv \Delta m_{41}^{2} L / 4 E$ ( $L$ being the baseline and $E$ the neutrino energy) is of order one, and the typical $L / E$ dependency of the signal is expected. However, we stress that sterile neutrinos can leave imprints also in other (non-short-baseline) kinds of experiments, where they have an impact in a more subtle way. In the atmospheric neutrinos, as first shown in [40], at $O(\mathrm{TeV})$ energies, a novel Mikheyev-Smirnov-Wolfenstein (MSW) resonance arises, which produces sizeable modifications of the zenith angle distribution. In the solar sector, a non-zero mixing of the electron neutrino with $v_{4}$ (parameterized by the matrix element $U_{e 4}$ ) leads to detectable deviations from the unitarity of the $\left(v_{1}, v_{2}, v_{3}\right)$ subsystem [36,37] (see also [38]).

Light sterile neutrinos can produce observable effects also in the long-baseline (LBL) accelerator experiments. We recall that such setups, when working in the $v_{\mu} \rightarrow v_{e}\left(\right.$ and $\bar{v}_{\mu} \rightarrow \bar{v}_{e}$ ) appearance channel, are able to identify the 3-flavor CP violation (CPV) induced by the standard CP-phase $\delta$. This sensitivity is strictly related to the circumstance that at long distances the $\nu_{\mu} \rightarrow v_{e}$ conversion probability contains an interference term (which is unobservable at SBL experiments) among the oscillations induced by the solar mass-squared difference and those driven by the atmospheric one. As first shown in [41], if sterile neutrinos participate in the oscillations, a new interference term arises in the conversion probability, which contains one additional CP-phase. Notably, the new genuine 4-flavor interference term is expected to have an amplitude similar to that of the standard 3-flavor interference term. Hence, the detection of the new interference term opens the concrete opportunity to explore the enlarged CPV sector implied by the presence of sterile neutrino species. It should be stressed that, in both the 3-flavor and 4-flavor frameworks, the CP-phases cannot be detected in SBL experiments, since in these setups the two standard frequencies $\Delta m_{21}^{2}$ and $\Delta m_{31}^{2}$ have a negligible impact. Therefore, the SBL and LBL facilities are synergic in searches of the $3+1$ framework (and of any other framework that involves more than one sterile state).

These basic observations provide the motivation for writing the present review, in which we discuss the physics potential of the future LBL experiments in the presence of a hypothetical light sterile neutrino. The subject is very active as testified by the numerous works about DUNE [42-48], T2HK [48-50], and T2HKK [48,51]. Other studies on the impact of light sterile neutrinos in LBL setups can be found in [52-60]. We underline that, while this review deals with charged current interactions, one can obtain valuable information on active-sterile oscillations parameters also from the analysis of neutral current interactions (see [21-24] for constraints from existing data and [47,61] for sensitivity studies of future experiments). Finally, we would like to stress that our review is limited to the oscillation phenomenology, although light sterile neutrinos may have an impact also on non-oscillation 
searches. We mention the studies focused on the leptonic decays of charged leptons and mesons [62], the beta decay [63], and the neutrinoless double-beta decay [64,65].

The review is organized as follows. In Section 2, we present the theoretical framework and introduce the basic formulae for the 4-flavor $v_{\mu} \rightarrow v_{e}$ and $\bar{v}_{\mu} \rightarrow \bar{v}_{e}$ transition probabilities. In Section 3, we provide the specifications of the three setups DUNE, T2HK, and ESS $v$ SB, which we use in the numerical simulations. In Section 4, we describe the details of the statistical method that we use for the analysis. In Section 5, we discuss the sensitivity to the LBL experiments to the NMH in the presence of a light sterile neutrino. In Section 6, we describe the sensitivity to CPV and, in Section 7, we study the ability to reconstruct the $\mathrm{CP}$ phases. In Section 8, we present an important degeneracy issue, which affects the reconstruction of the octant of $\theta_{23}$ in the $3+1$ scheme. We trace the conclusions in Section 9. Appendix A contains the analytical treatment of the matter effects relevant for LBL experiments in the $3+1$ scheme.

\section{Theoretical Framework}

In the presence of a sterile neutrino, the flavor $\left(v_{e}, v_{\mu}, v_{\tau}, v_{s}\right)$ and the mass eigenstates $\left(v_{1}, v_{2}, v_{3}, v_{4}\right)$ are connected by a $4 \times 4$ unitary matrix

$$
U=\tilde{R}_{34} R_{24} \tilde{R}_{14} R_{23} \tilde{R}_{13} R_{12},
$$

with $R_{i j}\left(\tilde{R}_{i j}\right)$ denoting a real (complex) $4 \times 4$ rotation of a mixing angle $\theta_{i j}$, which incorporates the $2 \times 2$ submatrix

$$
R_{i j}^{2 \times 2}=\left(\begin{array}{cc}
c_{i j} & s_{i j} \\
-s_{i j} & c_{i j}
\end{array}\right), \quad \tilde{R}_{i j}^{2 \times 2}=\left(\begin{array}{cc}
c_{i j} & \tilde{s}_{i j} \\
-\tilde{s}_{i j}^{*} & c_{i j}
\end{array}\right),
$$

in the $(i, j)$ sub-block. To simplify the notation, we define

$$
c_{i j} \equiv \cos \theta_{i j}, \quad s_{i j} \equiv \sin \theta_{i j}, \quad \tilde{s}_{i j} \equiv s_{i j} e^{-i \delta_{i j}} .
$$

The parameterization in Equation (1) enjoys some useful properties: (i) The 3-flavor matrix is recovered by setting $\theta_{14}=\theta_{24}=\theta_{34}=0$. (ii) For small values of the mixing angles $\theta_{14}, \theta_{24}$, and $\theta_{13}$, one has $\left|U_{e 3}\right|^{2} \simeq s_{13}^{2},\left|U_{e 4}\right|^{2}=s_{14}^{2},\left|U_{\mu 4}\right|^{2} \simeq s_{24}^{2}$, and $\left|U_{\tau 4}\right|^{2} \simeq s_{34}^{2}$, with a clear physical interpretation of the three mixing angles. (iii) The leftmost positioning of the matrix $\tilde{R}_{34}$ implies that the $v_{\mu} \rightarrow v_{e}$ transition probability in vacuum is independent of $\theta_{34}$ and of the associated CP phase $\delta_{34}$ (see [41]).

For clearness, we confine the discussion to the case of propagation in vacuum. We address the impact of matter effects in Appendix A. As first shown in [41], the $v_{\mu} \rightarrow v_{e}$ transition probability can be written as the sum of three contributions

$$
P_{\mu e}^{4 v} \simeq P^{\mathrm{ATM}}+P_{\mathrm{I}}^{\mathrm{INT}}+P_{\mathrm{II}}^{\mathrm{INT}} .
$$

The first term, which is positive-definite, depends on the atmospheric mass-squared splitting and provides the leading contribution to the probability. It takes the form

$$
P^{\mathrm{ATM}} \simeq 4 s_{23}^{2} s_{13}^{2} \sin ^{2} \Delta,
$$

where $\Delta \equiv \Delta m_{31}^{2} L / 4 E$ is the atmospheric oscillating factor, $L$ and $E$ being the neutrino baseline and energy, respectively. The other two contributions in Equation (4) arise from the interference of two different frequencies and are not positive-definite. Specifically, the second term in Equation (4) is related to the interference of the solar and atmospheric frequencies and can be written as

$$
P_{\mathrm{I}}^{\mathrm{INT}} \simeq 8 s_{13} s_{12} c_{12} s_{23} c_{23}(\alpha \Delta) \sin \Delta \cos \left(\Delta+\delta_{13}\right),
$$


where we introduce the ratio of the solar over the atmospheric mass-squared splitting, $\alpha \equiv \Delta m_{21}^{2} / \Delta m_{31}^{2}$. We recall that at the first (second) oscillation maximum one has $\Delta \sim \pi / 2(\Delta \sim 3 \pi / 2)$. The third contribution in Equation (4) appears as a new genuine 4-flavor effect, and is related to the interference of the atmospheric frequency with the new high frequency induced by the presence of the fourth mass eigenstate. This term can be written in the form [41]

$$
P_{\mathrm{II}}^{\mathrm{INT}} \simeq 4 s_{14} s_{24} s_{13} s_{23} \sin \Delta \sin \left(\Delta+\delta_{13}-\delta_{14}\right)
$$

From Equations (5)-(7), we see that the conversion probability depends on (besides the large mixing angle $\theta_{23}$ ) three small mixing angles: the standard angle $\theta_{13}$ and two new angles $\theta_{14}$ and $\theta_{24}$. We note that the values of such three mixing angles (estimated in the 3-flavor scenario [66-68] for $\theta_{13}$, and in the 4-flavor framework [12-15] for $\theta_{14}$ and $\theta_{24}$ ) are similar and one has $s_{13} \sim s_{14} \sim$ $s_{24} \sim 0.15$ (see Table 1 ). Therefore, one is naturally induced to consider these three angles as small quantities having the same order of magnitude $\epsilon$. We also notice that, since $|\alpha| \simeq 0.03$, it can be considered of order $\epsilon^{2}$. From inspection of Equations (5)-(7), we derive that the first (leading) contribution is of the second order, while the two interference terms are both of the third order. However, we underline that, differently from the standard interference term in Equation (6), the new sterile-induced interference term in Equation (7) is not proportional to $\Delta$ but to sin $\Delta$; hence, it is not enhanced at the second oscillation maximum. Because of this feature, as our numerical simulations will confirm, the performance of ESS $v$ SB in the 4-flavor scenario is not as good as that of DUNE and $\mathrm{T} 2 \mathrm{HK}$, which work around the first oscillation maximum.

Before closing this section, we underline the fact that the conversion probability in vacuum is independent of the third mixing angle $\theta_{34}$ and of the related CP-phase $\delta_{34}$. As shown in Appendix A, this conclusion does not hold anymore in the presence of matter. In practice, as we discuss in the subsequent sections, the CP-phase $\delta_{34}$ can be probed only in DUNE, which among the planned LBL experiments, is the most sensitive to the matter effects.

Table 1. Parameter values and ranges used in the simulations. The second column displays the true values of the oscillation parameters used to simulate the true dataset. The third column displays the range over which $\sin ^{2} \theta_{23}$ and the $C P$-phases $\delta_{13}, \delta_{14}$, and $\delta_{34}$ are varied while minimizing the $\chi^{2}$.

\begin{tabular}{ccc}
\hline Parameter & True Value & Marginalization Range \\
\hline $\sin ^{2} \theta_{12}$ & 0.304 & Not marginalized \\
$\sin ^{2} 2 \theta_{13}$ & 0.085 & Not marginalized \\
$\sin ^{2} \theta_{23}$ & 0.50 & {$[0.34,0.68]$} \\
$\sin ^{2} \theta_{14}$ & 0.025 & Not marginalized \\
$\sin ^{2} \theta_{24}$ & 0.025 & Not marginalized \\
$\sin ^{2} \theta_{34}$ & $0,0.025,0.25$ & Not marginalized \\
$\delta_{13} /^{\circ}$ & {$[-180,180]$} & {$[-180,180]$} \\
$\delta_{14} /{ }^{\circ}$ & {$[-180,180]$} & {$[-180,180]$} \\
$\delta_{34} /^{\circ}$ & {$[-180,180]$} & {$[-180,180]$} \\
$\frac{\Delta m_{21}^{2}}{10^{-5} \mathrm{eV}^{2}}$ & 7.50 & Not marginalized \\
$\frac{\Delta m_{31}^{2}}{10^{-3} \mathrm{eV}^{2}}(\mathrm{NH})$ & 2.475 & Not marginalized \\
$\frac{\Delta m_{31}^{2}}{10^{-3} \mathrm{eV}^{2}}(\mathrm{IH})$ & -2.4 & Not marginalized \\
$\frac{\Delta m_{41}^{2}}{\mathrm{eV}^{2}}$ & 1.0 & Not marginalized \\
\hline & &
\end{tabular}




\section{Description of the Experimental Setups}

\subsection{DUNE Setup}

Hosted at Fermilab, DUNE is a new-generation long-baseline neutrino oscillation experiment which will play an important role in the future neutrino roadmap to discover the fundamental properties of neutrino [69-73]. DUNE will be composed of three major ingredients: (a) an intense ( $\sim$ megawatt), broad-band neutrino beam at Fermilab; (b) a high-precision near neutrino detector just downstream of the neutrino source; and (c) a massive ( $\sim 40 \mathrm{kt})$ liquid argon time-projection chamber (LArTPC) far detector hosted deep underground at the Sanford Underground Research Facility (SURF) $1300 \mathrm{~km}$ away in Lead, South Dakota. We assume a fiducial mass of $35 \mathrm{kt}$ for the far detector in our simulations, and consider the detector properties which are given in Table 1 of Reference [74]. Concerning the beam, we consider a proton beam power of $708 \mathrm{~kW}$ with a proton energy of $120 \mathrm{GeV}$, which can deliver $6 \times 10^{20}$ protons on target on 230 days per calendar year. In our simulation, we have taken the fluxes which were estimated assuming a decay pipe length of $200 \mathrm{~m}$ and $200 \mathrm{kA}$ horn current [75]. We consider a total run time of ten years for this experiment, which is equivalent to a total exposure of $248 \mathrm{kt} \cdot \mathrm{MW} \cdot$ year. We assume that the DUNE experiment would use half of its full exposure in the neutrino mode, and the remaining half in the antineutrino mode. In our simulations, we take the reconstructed neutrino and anti-neutrino energy range to be $0.5-10 \mathrm{GeV}$. To incorporate the systematic uncertainties, we consider an uncorrelated $5 \%$ normalization error on signal and 5\% normalization error on background for both the appearance and disappearance channels to analyze the prospective data from the DUNE experiment. We consider the same set of systematics for both the neutrino and antineutrino channels which are also uncorrelated. For both $v_{e}$ and $\bar{v}_{e}$ appearance channels, the backgrounds mostly arise from three different sources: (a) the intrinsic $v_{e} / \bar{v}_{e}$ contamination of the beam; (b) the number of muon events which will be misidentified as electron events; and (c) the neutral current events. Our assumptions on various components of the DUNE set-up are similar to those mentioned in the Conceptual Design Report (CDR) reference design in Reference [70].

\subsection{T2HK Setup}

The main goal of the long-baseline neutrino program at the proposed Hyper-Kamiokande $(\mathrm{HK})$ detector with a neutrino beam from the J-PARC proton synchrotron is to give a conclusive evidence for leptonic CP-violation in neutrino oscillations induced by the CP-phase $\delta_{13}$ in the 3-flavor neutrino mixing matrix. This setup is commonly known as "T2HK" (Tokai to Hyper-Kamiokande) experiment [76-78]. To calculate the physics reach of this setup, we closely follow the experimental specifications as described in References [77,78]. The neutrino beam for HK will be produced at J-PARC from the collision of $30 \mathrm{GeV}$ protons on a graphite target. In our simulations, we consider an integrated proton beam power of $7.5 \mathrm{MW} \times 10^{7} \mathrm{~s}$, which can deliver in total $15.6 \times 10^{21}$ protons on target (p.o.t.) with a $30 \mathrm{GeV}$ proton beam. We assume that the T2HK experiment would use $25 \%$ of its full exposure in the neutrino mode which is $3.9 \times 10^{21}$ p.o.t. and the remaining $75 \%\left(11.7 \times 10^{21}\right.$ p.o.t. $)$ would be used for the antineutrino run. This ensures that we have nearly equal statistics in both neutrino and antineutrino modes to optimize the search for leptonic CP-violation. These neutrinos and antineutrinos will be observed in the gigantic $560 \mathrm{kt}$ (fiducial) HK water Cherenkov detector in the Tochibora mine, located $8 \mathrm{~km}$ south of Super-Kamiokande and $295 \mathrm{~km}$ away from J-PARC. The neutrino beamline from J-PARC is designed to produce an off-axis angle of $\sim 2.5^{\circ}$ at the proposed HK site and, therefore, the beam peaks at the first oscillation maximum of $0.6 \mathrm{GeV}$ to enhance the physics sensitivity. This off-axis scheme [79] gives rise to a neutrino beam with a narrow energy spectrum which substantially reduces the intrinsic $v_{e}$ contamination in the beam and also the background deriving from neutral current events. In our analysis, we consider the reconstructed neutrino and antineutrino energy range of $0.1-1.25 \mathrm{GeV}$ for the appearance channel. In case of disappearance channel, the assumed energy range is $0.1-7 \mathrm{GeV}$ for both the $v_{\mu}$ and $\bar{v}_{\mu}$ candidate events. We match all the signal and 
background event numbers following Tables 19 and 20 of Reference [77]. The systematic uncertainties play an important role in estimating the physics sensitivity of the T2HK setup. Following Table 21 of Reference [77], we consider an uncorrelated normalization uncertainty of $3.5 \%$ for both appearance and disappearance channels in neutrino mode. In the case of antineutrino run, the uncorrelated normalization uncertainties are $6 \%$ and $4.5 \%$ for appearance and disappearance channels, respectively, and they do not have any correlation with appearance and disappearance channels in neutrino mode. We assume an uncorrelated $10 \%$ normalization uncertainty on background for both appearance and disappearance channels in neutrino and antineutrino modes. With all these assumptions on the T2HK setup, we manage to reproduce all the sensitivity results which are given in Reference [77]. Here, we would like to mention that, according to the latest report by the Hyper-Kamiokande Proto-Collaboration [80], the total beam exposure is $27 \times 10^{21}$ p.o.t. and the fiducial mass for the proposed HK detector is $374 \mathrm{kt}$. Comparing the exposures in terms of $(\mathrm{kt} \times$ p.o.t.), we see that our exposure is 1.156 times smaller than the exposure that has been considered in Reference [80]. However, certainly, the results presented in this work would not change much and the conclusions drawn based on these results would remain valid even if we consider the new exposure as reported in Reference [80].

\subsection{ESSvSB Setup}

ESS $v B$ is a proposed superbeam on-axis experiment where a very high intense proton beam of energy $2 \mathrm{GeV}$ with an average beam power of $5 \mathrm{MW}$ will be delivered by the European Spallation Source (ESS) linac facility running at $14 \mathrm{~Hz}$. The number of protons on target (POT) per year (208 days) will be $2.7 \times 10^{23}$ [81-84]. It is worth mentioning here that the future linac upgrade can push the proton energy up to $3.6 \mathrm{GeV}$. The facility is expected to start taking neutrino data around 2030. We have obtained the fluxes from [85]. They peak around $0.25 \mathrm{GeV}$. A $500 \mathrm{kt}$ fiducial mass Water Cherenkov detector similar to the properties of the MEMPHYS detector $[86,87]$ is under investigation to explore the neutrino properties in this low energy regime. It has been shown in [81] that, if the detector is placed in any of the existing mines located within 300-600 km from the ESS site at Lund, it will make possible to reach $5 \sigma$ discovery of leptonic $\mathrm{CP}$-violation with $50 \%$ coverage of the whole range of the $\mathrm{CP}$ phase. A detailed study on the $\mathrm{CP}$-violation discovery capability of this facility with different baseline and different combinations of neutrino and antineutrino run time has also been performed in [88]. In this review, we consider a baseline of $540 \mathrm{~km}$ from Lund to Garpenberg mine located in Sweden and also we have matched the event numbers of Table 3 and all other results given in [81]. At this baseline, it works around the second oscillation maximum and it provides the opportunity to explore the $\mathrm{CP}$-asymmetry, which (in the 3-flavor scheme) is three times larger than the CP-asymmetry at the first oscillation maximum. Although the main disadvantages for going to the second oscillation maximum come from the significant decrease of statistics and cross-sections compared to the first oscillation maximum, the intense beam of this facility compensates those difficulties and makes the statistics competitive to provide interesting results. All our simulations presented here for this setup have been done assuming two years of $v$ and eight years of $\bar{v}$ running. We set the uncorrelated $5 \%$ signal normalization and 10\% background normalization error for both neutrino and antineutrino appearance and disappearance channels, respectively. For more details of the accelerator facility, beamline design, and detector facility of this setup please, see Reference [81].

\section{Details of the Statistical Analysis}

The experimental sensitivities presented in this review are taken form the works $[45,50,89]$. These have been calculated making use of the GLoBES software $[90,91]$ together with its new physics package. The sterile neutrino effects are included both in the $v_{\mu} \rightarrow v_{e}$ appearance channel and in the $v_{\mu} \rightarrow v_{\mu}$ disappearance channel. The same holds for antineutrino mode. Table 1 displays the true values of the oscillation parameters and their respective ranges of marginalization, which we consider in our numerical simulations. Apart from the atmospheric mixing angle $\theta_{23}$, we have fixed the 3-flavor 
oscillation parameters equal to those obtained in the most recent global fits [66-68]. For $\theta_{23}$ (apart from Section 8$)$, we have set its value to be maximal $\left(45^{\circ}\right)$, and in the fit we have marginalized it over the range indicated in Table 1 . Concerning the mixing angles that involve the fourth state $\left(\theta_{14}, \theta_{24}\right.$, and $\left.\theta_{34}\right)$, we have fixed both their true and fit values as given in Table 1 . For $\sin ^{2} \theta_{34}$, we consider three options, namely $0,0.025$, and 0.25 , which are within its current allowed range [12-15]. Concerning the $C P$ phases $\delta_{14}$ and $\delta_{34}$, we vary their true values in the range $[-\pi, \pi]$, and marginalize the fit values in the same range. We set the mass-squared splitting to $\Delta m_{41}^{2}=1 \mathrm{eV}^{2}$, which is the value presently indicated by the SBL data. However, we underline that our findings would be unaltered for different values of such a parameter, provided that $\Delta m_{41}^{2}>0.1 \mathrm{eV}^{2}$. For such values, the fast oscillations induced by the large mass-squared splitting are totally averaged by the finite energy resolution of the detector. For the same motivation, LBL setups are insensitive to the sign of $\Delta m_{41}^{2}$ and this allows us to limit our study to positive values. We have set the line-averaged constant Earth matter density ${ }^{2}$ of $2.87 \mathrm{~g} / \mathrm{cm}^{3}$ for all the LBL experiments considered. In our simulations, we have incorporated a full spectral analysis by making use of the binned events spectra for the each LBL experiment. In the statistical analysis, the Poissonian $\Delta \chi^{2}$ has been marginalized over the systematic uncertainties using the pull method as prescribed in References [93,94]. For displaying the results, we show the 1,2,3 $\sigma$ confidence levels for 1 d.o.f. adopting the relation $\mathrm{N} \sigma \equiv \sqrt{\Delta \chi^{2}}$.

In [95], it was shown that the above expression holds in the frequentist method of hypothesis testing. We define the $\chi^{2}$ function

$$
\chi^{2}=\min _{\tilde{\xi}_{s}, \tilde{\xi}_{b}}\left[2 \sum_{i=1}^{n}\left(\tilde{y}_{i}-x_{i}-x_{i} \ln \frac{\tilde{y}_{i}}{x_{i}}\right)+\xi_{s}^{2}+\xi_{b}^{2}\right],
$$

where $n$ is the total number of reconstructed energy bins and

$$
\tilde{y}_{i}\left(\{\omega\},\left\{\xi_{s}, \xi_{b}\right\}\right)=N_{i}^{t h}(\{\omega\})\left[1+\pi^{s} \xi_{s}\right]+N_{i}^{b}(\{\omega\})\left[1+\pi^{b} \xi_{b}\right] .
$$

Above, $N_{i}^{\text {th }}(\{\omega\})$ represents the theoretical number of CC signal events in the $i$ th energy bin for a set of oscillation parameters $\omega . N_{i}^{b}(\{\omega\})$ denotes the total number of background events ${ }^{3}$. The quantities $\pi^{s}$ and $\pi^{b}$ in Equation (9) denote the systematic normalization errors on the signal and background events, respectively. We take the same uncorrelated systematic error for both the neutrino and antineutrino channels. The quantities $\xi_{s}$ and $\xi_{b}$ denote the "pulls" due to the systematic error on the signal and background, respectively. The data in Equation (8) are included through the variable $x_{i}=N_{i}^{e x}+N_{i}^{b}$, where $N_{i}^{e x}$ is the number of observed CC signal events and $N_{i}^{b}$ is the background as discussed above. To obtain the total $\chi^{2}$, we add the $\chi^{2}$ contributions coming from all the relevant oscillation channels for both neutrino and antineutrino modes in a given experiment

$$
\chi_{\text {total }}^{2}=\chi_{v_{\mu} \rightarrow v_{e}}^{2}+\chi_{\bar{v}_{\mu} \rightarrow \bar{v}_{e}}^{2}+\chi_{v_{\mu} \rightarrow v_{\mu}}^{2}+\chi_{\bar{v}_{\mu} \rightarrow \bar{v}_{\mu}}^{2} .
$$

In the above expression, we assume that all the oscillation channels for both neutrino and antineutrino modes are uncorrelated, all the energy bins in a given channel are fully correlated, and the systematic errors on signal and background are uncorrelated. The fact that the flux normalization errors in $v_{\mu} \rightarrow v_{e}$ and $v_{\mu} \rightarrow v_{\mu}$ oscillation channels are the same (i.e., they are correlated) is taken into account in the error budget for each of the two channels. However, there are other uncertainties which contribute to the global normalization error for each of the two channels, such as the uncertainties in

2 The line-averaged constant Earth matter density has been computed using the Preliminary Reference Earth Model (PREM) [92].

3 Note that we consider both CC and NC background events in our analysis and the NC background is independent of oscillation parameters. 
cross sections, detector efficiencies, etc., which are uncorrelated. For this reason, we simply assume that the total normalization errors in these two channels are uncorrelated. The same holds for $\bar{v}_{\mu} \rightarrow \bar{v}_{e}$ and $\bar{v}_{\mu} \rightarrow \bar{v}_{\mu}$ oscillation channels. We think that, with the current understanding of the detectors of DUNE, T2HK, and ESS $v$ SB experiments, it is premature to perform a more accurate analysis taking into account more fine effects as, e.g., the correlation between the flux normalization errors in the appearance and disappearance channels.

\section{Mass Hierarchy Discovery Potential in the $3+1$ Scheme}

In this section, we present the discovery potential of the future LBL experiments to the neutrino mass hierarchy $(\mathrm{NMH})$. We recall that the $\mathrm{NMH}$ discovery potential of a given experiment is proportional to the size of matter effects in that setup. This in turn is related to the energy of the neutrino beam of the experiment. As a result, DUNE is very sensitive to NMH, working at the energy of $E=2.5 \mathrm{GeV}$; T2HK has intermediate sensitivity since it works at $E=0.6 \mathrm{GeV}$; and ESS $2 \mathrm{SB}$ has basically no sensitivity working at $E=0.25 \mathrm{GeV}$. In the following we show the sensitivity of DUNE and $\mathrm{T} 2 \mathrm{HK}$ in the 3-flavor and 4-flavor schemes.

In Figure 1, we present the bi-event plots, in which the two axes report the total number of $v_{e}$ $\left(x\right.$-axis) and $\bar{v}_{e}$ (y-axis) events ${ }^{4}$. In both panels, the black ellipses represent the 3 -flavor case, and are obtained by varying the CP-phase $\delta_{13}$ in the range $[-\pi, \pi]$. In the $3+1$ scheme, two CP-phases are present and the bi-event plot becomes a blob. In both panels, we assume $\theta_{14}=\theta_{24}=9^{\circ}$, and $\theta_{34}=0$, varying the two phases $\delta_{13}$ and $\delta_{14}$ in the range $[-\pi, \pi]$. The 4 -flavor full regions can be regarded as a convolution of an ensemble of ellipses with different orientations or, alternatively, as a dense scatter plot resulting from the simultaneous variation of the CP-phases $\delta_{13}$ and $\delta_{14}$. One can see that, in the left panel concerning DUNE, there is a neat separation between the 3-flavor ellipses. This guarantees and exceptional sensitivity of DUNE to the NMH in the standard 3-flavor scheme. In the $3+1$ scenario, the separation among the blobs corresponding to the two hierarchies is reduced in comparison to the 3-flavor ellipses, but still the two hierarchies remain distinct. Therefore, we expect that the NMH discovery potential will be reduced in the $3+1$ scheme, but it will not be completely erased. In the right panel, we present the same kind of plot concerning T2HK. In this case, already at the level of the 3-flavor scenario we expect a low sensitivity to $\mathrm{NMH}$ since the $\mathrm{NH}$ and $\mathrm{IH}$ ellipses overlap. In the $3+1$ scheme, the level of overlapping remains similar.

In Figure 2, we display the discovery potential of the correct hierarchy as a function of the true value of $\delta_{13}$ considering $\mathrm{NH}$ as the true choice. The left (right) panel refers to DUNE (T2HK). In each panel, we provide the results for the 3-flavor case, which is represented by a black curve. In both panels, for the 4-flavor scenario, we have taken $\theta_{14}=\theta_{24}=9^{\circ}$ and $\theta_{34}=0$, both true and fit values. Concerning DUNE (left), we present the results for four values of the true $\delta_{14}\left(-90^{\circ}, 90^{\circ}, 0^{\circ}\right.$, and $\left.180^{\circ}\right)$ while we marginalize the fit value of $\delta_{14}$ in the range of $[-\pi, \pi]$. Qualitatively, the shape of the $3+1$ curves seems similar to the 3-flavor one. In particular, a maximum is present around $\delta_{13} \sim-90^{\circ}$, and a minimum around $\delta_{13} \sim 90^{\circ}$. It is evident that overall, there is a deterioration of the sensitivity of DUNE for all values of the new CP-phase $\delta_{14}$. However, even in the region around the minimum, the sensitivity never decreases below the $5 \sigma$ level. In the right panel of Figure 2, we plot the NMH discovery potential for T2HK. In the 3-flavor case, on the basis of the bivents plot, one would expect that for some values of the phase $\delta_{13}$, the sensitivity should drop to zero. However, quite surprisingly, a non-zero sensitivity appears. As extensively discussed in [50], the sensitivity is due to the energy spectral information. The green band in the right panel represents the T2HK sensitivity in the $3+1$ scheme. The width of the band is due to the spanning of the true value of $\delta_{14}$ of the range of $[-\pi, \pi]$. We notice that the shape of the $3+1$ band is similar to that of the 3-flavor scenario, and, as expected from the bi-event plot, there is only a weak degradation of the $\mathrm{NMH}$ discovery potential. In addition,

4 In the 3+ 1 scheme, these plots were first introduced in Reference [96] for the discussion of $\mathrm{T} 2 \mathrm{~K}$ and NO$v \mathrm{~A}$. 
in this case, the binned spectrum has a key role in the NMH discrimination. We can conclude that, albeit the NMH sensitivity of T2HK is quite limited, it is robust with respect to the modifications produced by a light sterile neutrino state.
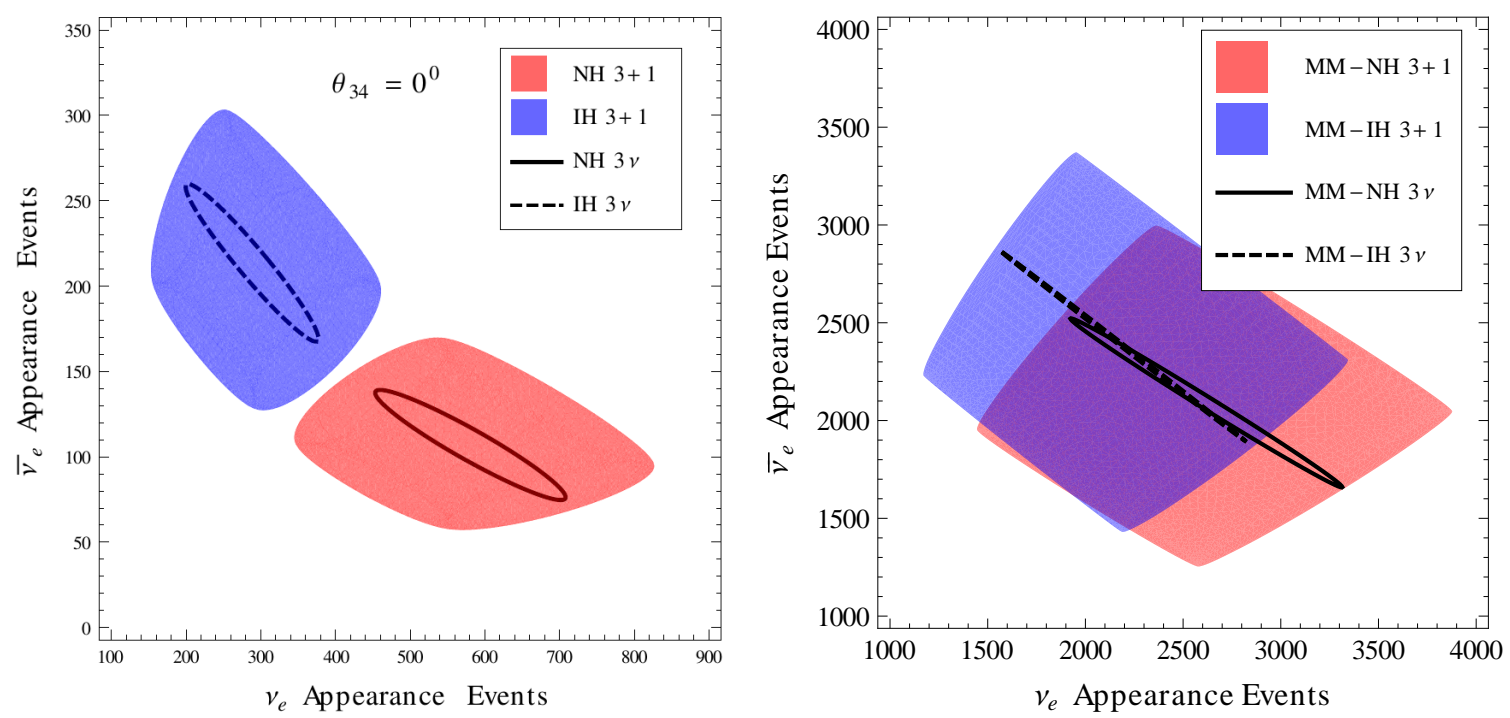

Figure 1. The colored blobs in the left (right) panel represent the bi-event plots for DUNE (T2HK) in the $3+1$ scenario. The regions are obtained by varying the CP-phases $\delta_{13}$ and $\delta_{14}$ in the range $[-\pi, \pi]$. The black curves represents the 3-flavor ellipses. In this case, there is only one running parameter, the CP-phase $\delta_{13}$. Both figures are obtained for maximal $\theta_{23}$ mixing. This is explicitly indicated only in the right panel as "MM" in the legend. Figures taken from [45] (left) and [50] (right).
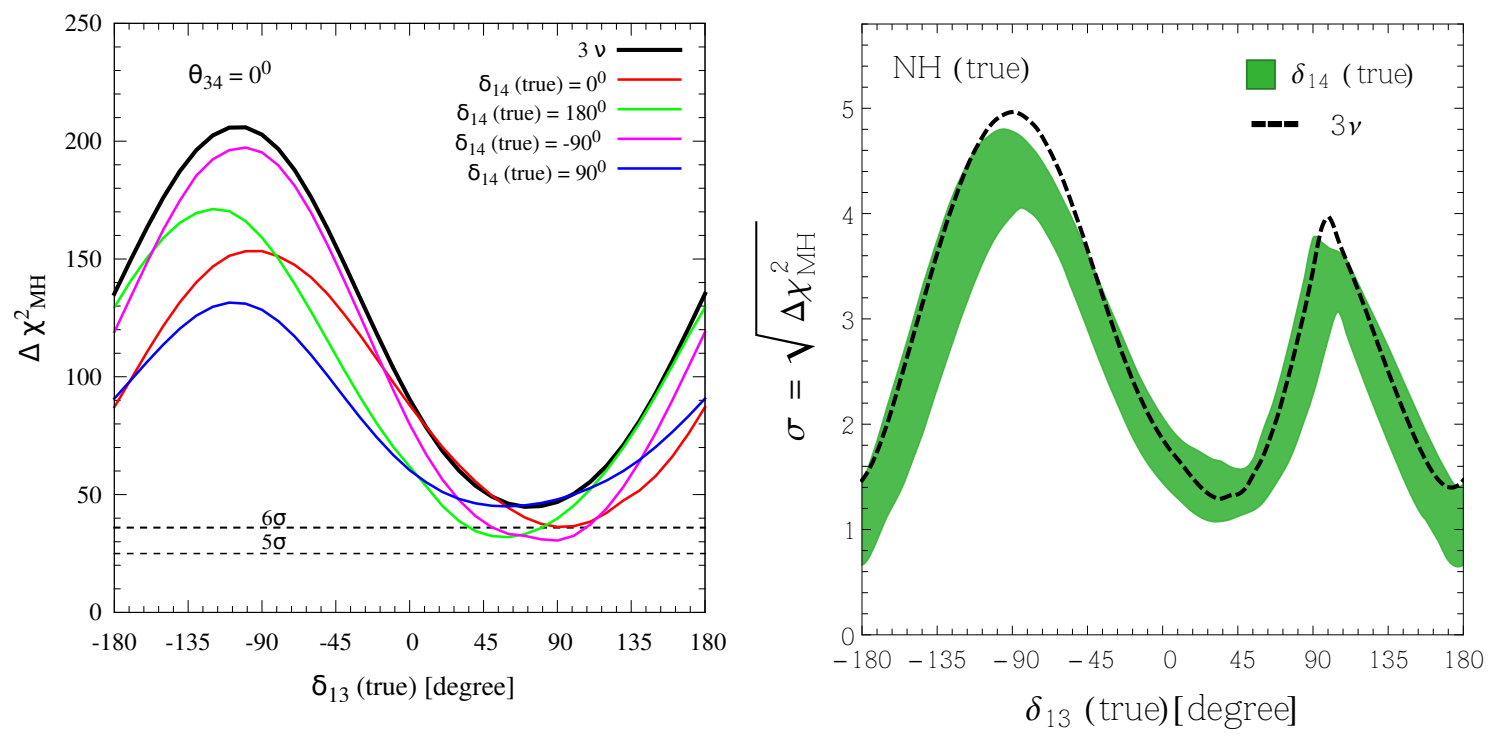

Figure 2. Discovery potential of DUNE (left) and T2HK (right) for identifying the correct hierarchy $(\mathrm{NH})$ as a function of true $\delta_{13}$. In both panels, we have fixed true and test $\theta_{14}=\theta_{24}=9^{\circ}$ and $\theta_{34}=0$. In both panels, the black curve represents the 3 -flavor case. For DUNE (left), in the $3+1$ scheme, we provide the curves for four different values of true $\delta_{14}$ marginalizing over test $\delta_{14}$. For T2HK (right), the green band is obtained by varying the true value of $\delta_{14}$ in the range $[-\pi, \pi]$ and marginalizing the test value of $\delta_{14}$ in the same range. Figures taken from [45] (left) and [50] (right). 


\section{CP-violation Discovery Potential}

The sensitivity of CPV induced by a given (true) value of a CP phase $\delta_{i j}^{\text {true }}$ is defined as the statistical significance at which one can exclude the test hypothesis of no CPV, i.e., the (test) cases $\delta_{i j}^{\text {test }}=0$ and $\delta_{i j}^{\text {test }}=\pi$. In Figure 3, we report the discovery potential of CPV induced by $\delta_{13}$ for DUNE (left), T2HK (middle), and ESS $v$ SB (right). We have assumed that the hierarchy is known a priori and is $\mathrm{NH}$. In all panels, the black dashed curve corresponds to the 3 -flavor scenario while the colored band represents the $3+1$ scheme. In the $3+1$ scenario, we fix the true and test values of $\theta_{14}$ and $\theta_{24}$ to be $9^{\circ}$. In all panels, the colored bands are obtained by varying the unknown true value of $\delta_{14}$ in the range of $[-\pi, \pi]$ while marginalizing over its test value in the same range. We can observe that in all cases, in the $3+1$ scheme, there is a non-negligible deterioration of the sensitivity. The deterioration is more pronounced in ESSvSB (right). As explained in detail in [89], this different behavior is related to the fact that ESSvSB (in the configuration which we are considering, corresponding to a baseline of $540 \mathrm{~km}$ ) works at the second oscillation maximum, in contrast to DUNE and T2HK which work around the first oscillation maximum.
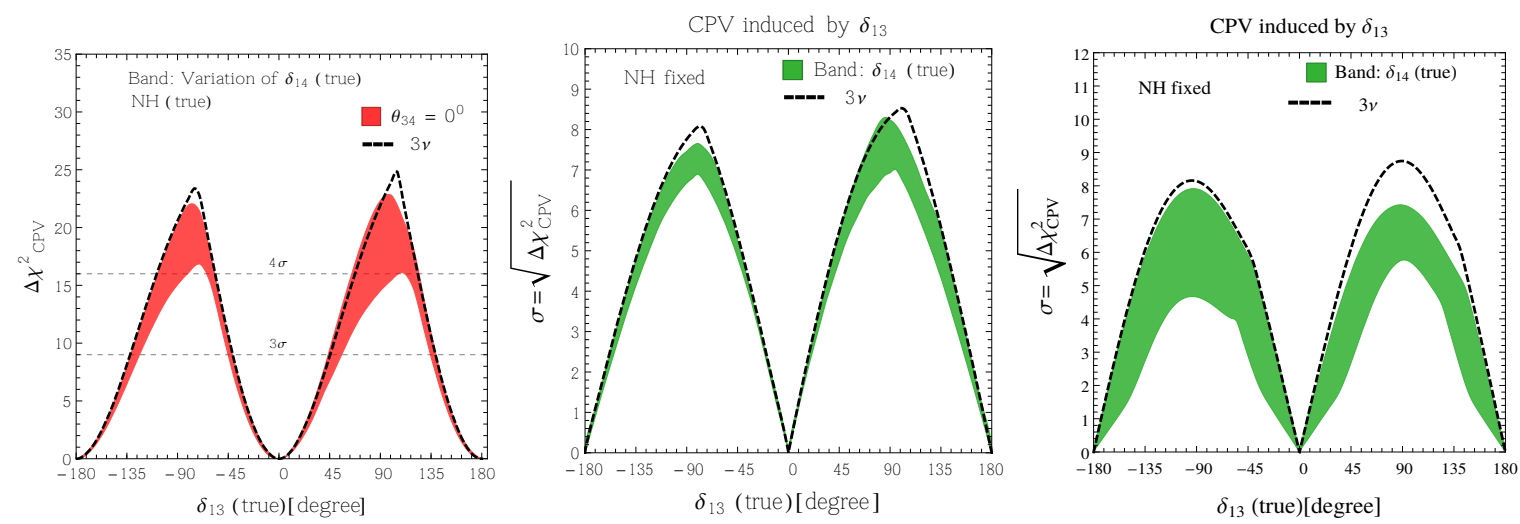

Figure 3. Sensitivity to CPV induced by $\delta_{13}$ for DUNE (left), T2HK (central), and ESS $\mathrm{SB}$ (right) assuming the $\mathrm{NMH}$ is known to be the $\mathrm{NH}$. In all three panels, the black dashed curve corresponds to the 3-flavor result. In the 4-flavor scheme, we fix the true and test values of $\theta_{14}=\theta_{24}=9^{\circ}$, and we take the true and test $\theta_{34}$ to be $0^{\circ}$. In all panels, the colored bands are obtained in the $3+1$ scheme by varying the unknown true $\delta_{14}$ in the range of $[-\pi, \pi]$ in the data while marginalizing over fit $\delta_{14}$ in the same range. Figures taken from [45] (left), [50] (central), and [89] (right).

In the $3+1$ scheme, one expects that CPV may arise also from the two new phases $\delta_{14}$ and $\delta_{34}$. However, a non-zero sensitivity to $\delta_{34}$, can be obtained only in DUNE, where matter effects are noticeable, and for relatively big values of the mixing angle $\theta_{34}$. For this reason, we first show the discovery potential of the two experiments T2HK and ESS $v$ SB, which are only sensitive to $\delta_{14}$. In the left and right panels of Figure 4, we display the discovery potential of the CPV induced by $\delta_{14}$ in T2HK and ESS $v S B$, respectively. We observe that the T2HK performance is much better than that of ESS $v$ SB. In the first case one can reach a sensitivity of $5 \sigma$, while in the second one the sensitivity never exceeds the $2 \sigma$ level. 

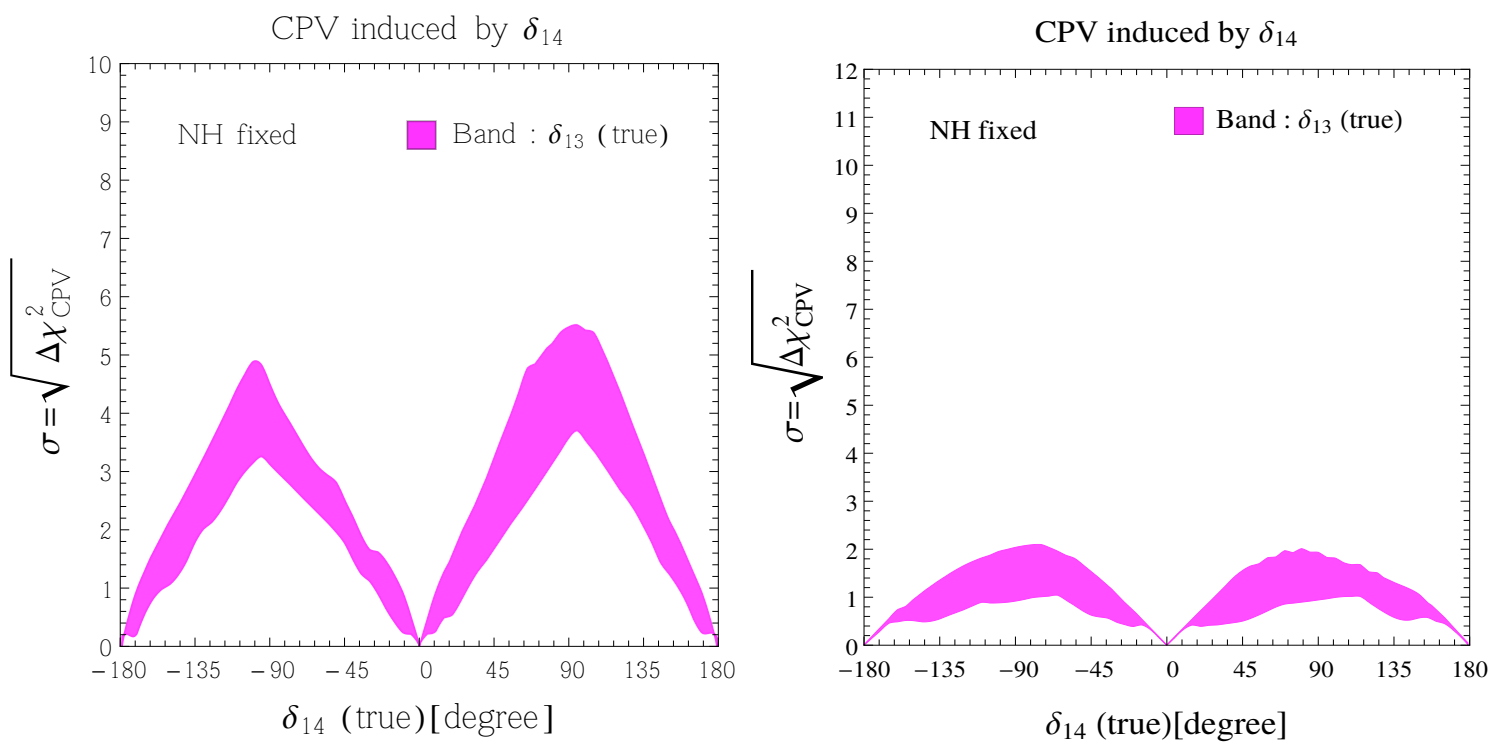

Figure 4. Discovery potential of the CPV induced by $\delta_{14}$ for T2HK (left) and ESS 2 SB (right), assuming the $\mathrm{NMH}$ is known to be the $\mathrm{NH}$. We fix the true and test values of $\theta_{14}=\theta_{24}=9^{\circ}$, and we take the true and test $\theta_{34}$ to be $0^{\circ}$. In both panels, the colored bands are obtained in the $3+1$ scheme by varying the unknown true $\delta_{14}$ in its entire range of $[-\pi, \pi]$ in the data while marginalizing over test $\delta_{14}$ in the same range. Figures taken from [50] (left) and [89] (right).

We think that it is useful to further comment on this huge difference in the sensitivity to CPV induced by $\delta_{14}$ between the two experiments ESS $v$ SB and T2HK. We notice (see Figure 3 ) that in the 3-flavor framework both experiments have comparable sensitivities to CPV driven by $\delta_{13}$, having both the highest sensitivity of about $8 \sigma$ for $\delta_{13} \simeq \pm 90^{\circ}$. This is possible, because, despite the lower statistcal power, ESS $v$ SB takes advantage of the amplification factor proportional to the atmospheric oscillating factor $\Delta$, which appears in the standard 3-flavor interference term [see Equation (6)] of the conversion probability. In fact, this is approximately three times bigger around the second oscillation maximum with respect to the first one. In contrast, in the $3+1$ scheme, the sensitivity is much worse in ESS $v$ SB. In fact, one can observe that: (i) The degradation of the discovery potential to the CPV induced by $\delta_{13}$ when going from the 3-flavor to the $3+1$ framework is much more noticeable in ESS vSB than in T2HK. Taking the values $\delta_{13}= \pm 90^{\circ}$ as a benchmark (where the highest sensitivity is reached), we find for T2HK only a weak deterioration of the discovery potential from $8 \sigma$ to $7 \sigma$ (see middle panel in Figure 3). In ESS $v$ SB, we find instead a pronounced reduction from $8 \sigma$ to $4.5 \sigma$ (see right panel in Figure 3); (ii) The sensitivity to the CPV driven by the new CP phase $\delta_{14}$ is much lower in ESS $v$ SB than in T2HK ( $2 \sigma$ vs. $5 \sigma$ for $\delta_{14}= \pm 90^{\circ}$ ). The explanation of such a different performance in the $3+1$ scheme of the two experiments can be understood noticing the fact that ESS $v$ SB works at the second oscillation maximum. As already stressed in Section 2, the new interference term (which is sensitive to $\delta_{14}$ ) depends on $\sin \Delta$ [see Equation (6)], and at the second oscillation maximum it is not amplified by the factor $\Delta$ as happens for the standard 3-flavor interference term (which is sensitive to $\delta_{13}$ ). In addition, as underlined in [50], T2HK can benefit from the energy spectral information, which plays a crucial role in maintaining a good performance in the $3+1$ scheme. Indeed, in [50], it was demonstrated that, even if there is a degeneracy at the level of the event counting, the energy spectrum is able to break such a degeneracy, thus boosting the sensitivity. In ESSvSB, the impact of the spectral information is drastically reduced because the energy range at the second oscillation maximum is very narrow and the low statistics impedes to use the information contained in the spectrum. Therefore, we conclude that ESS $v$ SB is not particularly suited for the searches of CPV induced by the presence of sterile neutrinos. We underline that such a conclusion holds for a baseline of $540 \mathrm{~km}$, for which ESS $v$ SB works around the second oscillation maximum. Things may improve by choosing a smaller baseline of $360 \mathrm{or} 200 \mathrm{~km}$. In this last case, ESS $v$ SB would work around the first oscillation maximum. 
Now, let us come to the sensitivity or DUNE to the new CP-phases, which is depicted in Figure 5. In the first panel, for comparison, we display the sensitivity to CPV induced by the standard $\mathrm{CP}$-phase $\delta_{13}$. The thinner (magenta) bands represent to the case in which all the three new mixing angles have the same values $\theta_{14}=\theta_{24}=\theta_{34}=9^{\circ}$. The thicker (green) bands correspond to the case in which $\theta_{14}=\theta_{24}=9^{\circ}$, and $\theta_{34}=30^{\circ}$. In each panel, the bands have been obtained by varying the true values of the two undisplayed CP-phases in their allowed ranges of $[-\pi, \pi]$ in the data while marginalizing over their fit values in the same range. The comparison of the three panels show that, if all the three mixing angles have the same value $\theta_{14}=\theta_{24}=\theta_{34}=9^{\circ}$, there is a clear hierarchy in the sensitivity to the three $\mathrm{CP}$-phases. The $\mathrm{CP}$ phase $\delta_{13}$ comes first, $\delta_{14}$ comes next, and $\delta_{34}$ is the last one, inducing negligible CPV. Concerning the CP-phase $\delta_{14}$ (middle), the sensitivity spans over a wide band. In favorable cases, the signal lies above the $3 \sigma$ level. On the other hand, the lower border of the band is always below the $\sim 2 \sigma$. This means that there is not a guaranteed discovery potential at the $3 \sigma$ confidence level for CPV induced by $\delta_{14}$. Finally, coming to the third CP-phase $\delta_{34}$ (right), we observe that, if the mixing angle $\theta_{34}$ is big, the sensitivity can be noticeable. In addition, we see that the shape of the band is different from that obtained for the other two CP-phases. This different behavior can be explained by observing that, in this case, the $v_{\mu} \rightarrow v_{\mu}$ channel also contributes to the overall sensitivity. In fact, it is well known that the $v_{\mu}$ survival probability has a good sensitivity to the NSI-like coupling $\varepsilon_{\mu \tau}$. From the expression of the Hamiltonian in Equation (A13), one can observe that the new mixing angles mimic a NSI-like perturbation $\varepsilon_{\mu \tau}=r s_{24} \tilde{S}_{34}^{*}$, thus a sensitivity of the $v_{\mu}$ survival probability to the CP-phase $\delta_{34}$ is expected.

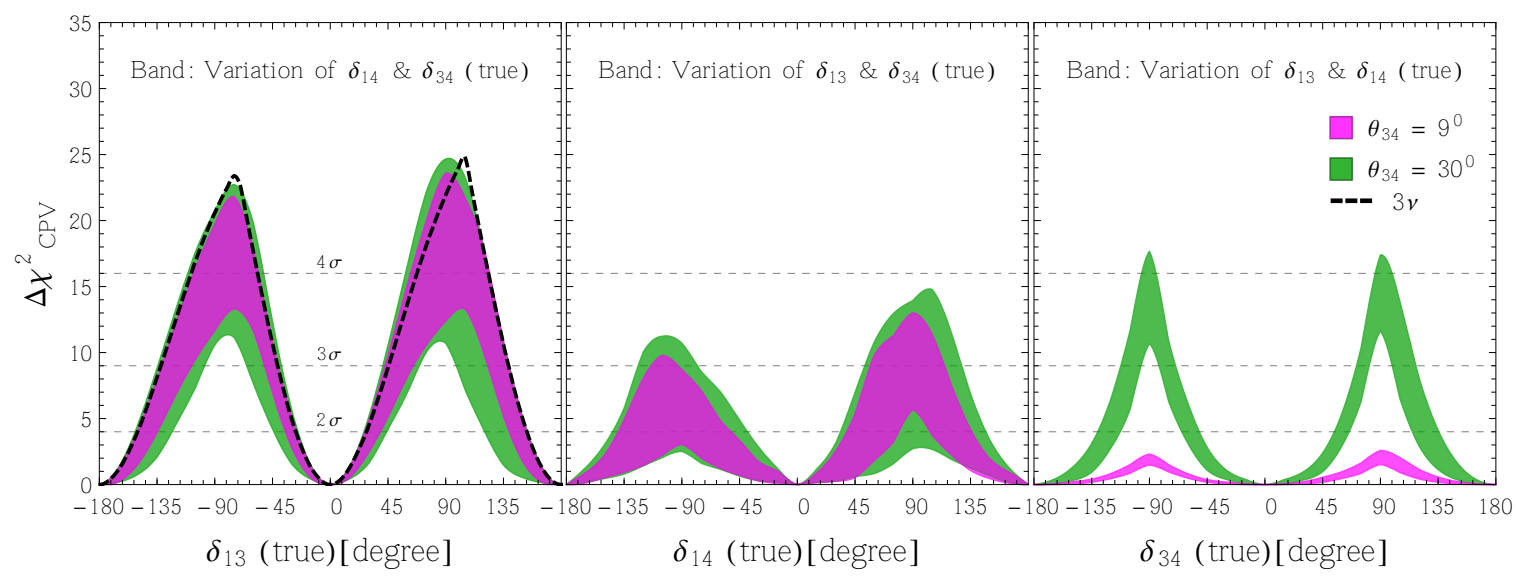

Figure 5. The left, central, and right panels display the discovery potential of the CPV induced, respectively, by $\delta_{13}, \delta_{14}$, and $\delta_{34}$ in the $3+1$ scenario. The thinner (magenta) bands correspond to the case in which the three new angles have the same value $\theta_{14}=\theta_{24}=\theta_{34}=9^{\circ}$. The thicker (green) bands correspond to the case in which $\theta_{14}=\theta_{24}=9^{\circ}$, and $\theta_{34}=30^{\circ}$. In each panel, the bands have been obtained by varying the true values of the two undisplayed CP-phases in the range $[-\pi, \pi]$. The left panel also reports the 3-flavor curve (black dashed line) for comparison. In all cases, we marginalize over hierarchy with $\mathrm{NH}$ taken as as the true choice. Figure taken from [45].

\section{Reconstruction of the CP Phases}

Until now, we have considered the discovery potential to the CPV induced by the two CP phases $\delta_{13}$ and $\delta_{14}$. Here, we study the capability of the LBL setups to reconstruct the true value of the two CP phases. With this purpose, we consider the four benchmark cases shown in Figure 6 . In each panel, we show the region reconstructed by each of the three LBL experiments, DUNE, T2HK, and ESSvSB, around a pair of true values of the two CP-phases $\delta_{13}$ and $\delta_{14}$. The first two panels represent the CP-conserving cases $(0,0)$ and $(\pi, \pi)$. The lower panels concern two CP-violating scenarios $(-\pi / 2,-\pi / 2)$ and $(\pi / 2, \pi / 2)$. In each panel, we show the regions reconstructed around the true values of the two $\mathrm{CP}$ phases. In these plots, we have fixed the $\mathrm{NH}$ as the true and test hierarchy. 
The contours are displayed for the $3 \sigma$ level (1 d.o.f.) ${ }^{5}$. The performance of ESS $v$ SB in reconstructing $\delta_{13}$ is similar to that of DUNE and T2HK. In contrast, the reconstruction of $\delta_{14}$ is better in T2HK and DUNE.
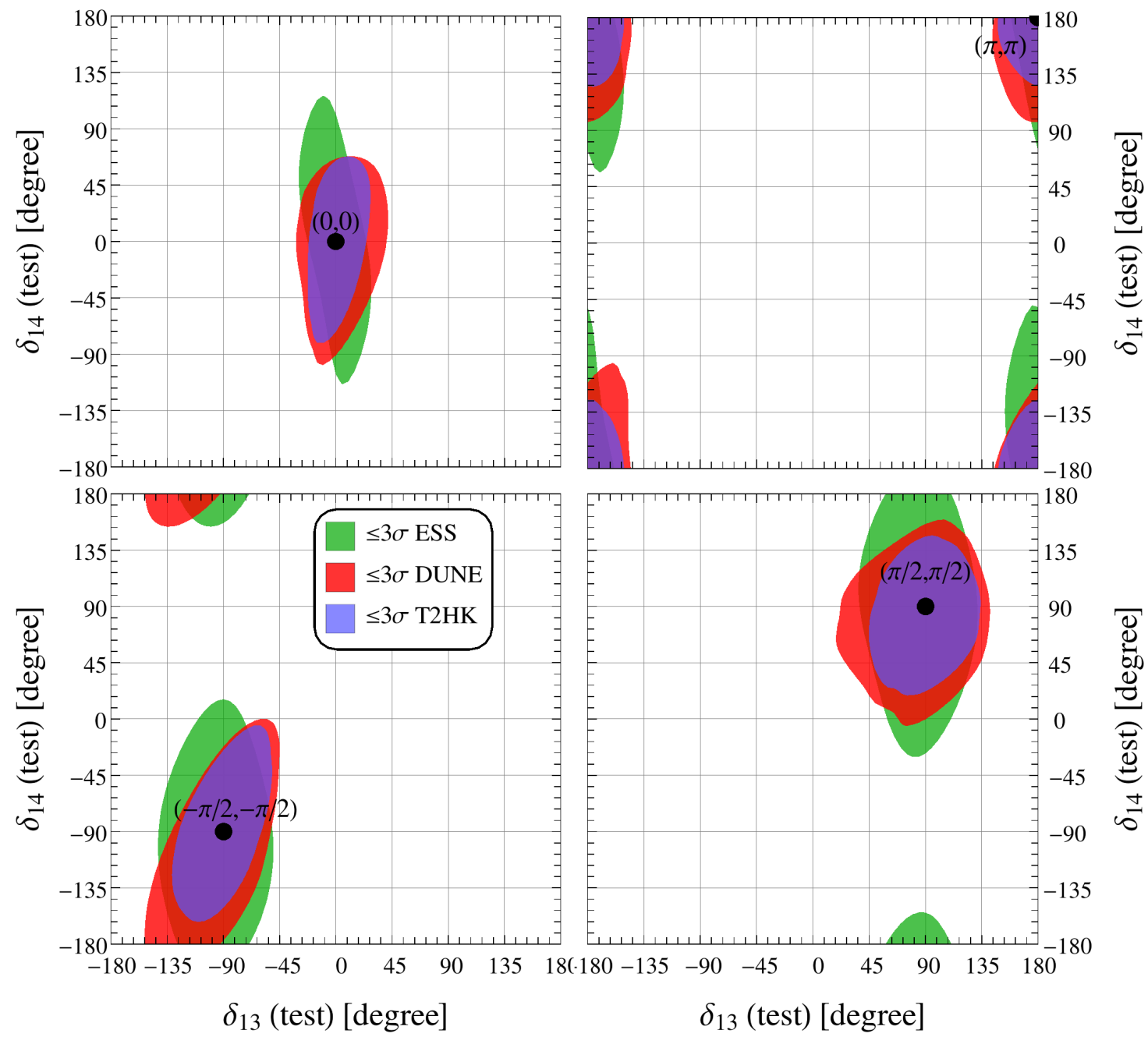

Figure 6. Reconstructed regions for the two CP phases $\delta_{13}$ and $\delta_{14}$ for the four representative couples of their true values indicated in each panel. Results are displayed for the three different experiments: ESS $v$ SB, DUNE, and T2HK. The NH has been fixed as the true and test hierarchy. The regions are drawn for the $3 \sigma$ (1 d.o.f.) confidence level. Figure taken from [89].

\section{Sterile Neutrinos and the Octant of $\theta_{23}$}

The latest global neutrino oscillation data fits favor a non-maximal value of the atmospheric mixing angle $\theta_{23}$ with two almost degenerate solutions: one $<\pi / 4$, denoted as lower octant (LO), and the other $>\pi / 4$, defined as higher octant $(\mathrm{HO})$. The identification of the $\theta_{23}$ octant is an important target in neutrino physics because of its deep ramifications in the theory of neutrino mass model building (see [97-101] for reviews). Notable models where the $\theta_{23}$ octant has an important role are

5 Note that both $\delta_{13}$ and $\delta_{14}$ are cyclic variables. Therefore, the union of the four corners in the top right panel of Figure 6 gives rise to a single connected region. 
$\mu \leftrightarrow \tau$ symmetry $^{6}$ [104-111], $A_{4}$-flavor symmetry [112-116], quark-lepton complementarity [117-120], and neutrino mixing anarchy $[121,122]$. From a phenomenological viewpoint, the information on the $\theta_{23}$ octant is also a key input. In fact, it is widely recognized that the identification of the two unknown properties (NMH and $\mathrm{CPV}$ ) is strictly entangled with the determination of $\theta_{23}$ due to parameter degeneracy problems [123-131].

The LBL experiments offer a unique opportunity to identify the $\theta_{23}$ octant. In fact, a well-known synergy between the $v_{\mu} \rightarrow v_{e}$ appearance and $v_{\mu} \rightarrow v_{\mu}$ disappearance channels [123,126] confers a high sensitivity to the $\theta_{23}$ octant. The $v_{\mu} \rightarrow v_{\mu}$ survival probability depends on $\sin ^{2} 2 \theta_{23}$. Then, it is sensitive to deviations from maximal $\theta_{23}$ but is not sensitive at all to its octant. In contrast, the $v_{\mu} \rightarrow v_{e}$ probability is proportional to $\sin ^{2} \theta_{23}$ and is sensitive to the octant. Therefore, the two channels give complementary information on $\theta_{23}$. Notably, the sensitivity can be maximized with a balanced exposure of neutrino and antineutrino runs [128].

Several sensitivity studies to the octant of $\theta_{23}$ have been performed within the 3-flavor scenario (see [128,132-136]). In the work [46], the issue has been studied in the $3+1$ scheme, evidencing for the first time an important degeneracy problem taking DUNE as a case study. We refer the reader to work [46] for analytical details, showing here the numerical results. In a nutshell, the newly identified interference term in Equation (7) that appears in the $v_{\mu} \rightarrow v_{e}$ transition probability in Equation (4) can mimic an inversion of the $\theta_{23}$ octant. As a consequence, for unlucky combinations of the two CP-phases $\delta_{13}$ and $\delta_{14}$, in the $3+1$ scheme, the discovery potential of the octant of $\theta_{23}$ is completely lost. The bi-event plot in Figure 7 provides a bird-eye view of what happens. In the $3+1$ scheme, the theoretical prediction is represented by a colored blob, and the separation among LO and HO is lost.

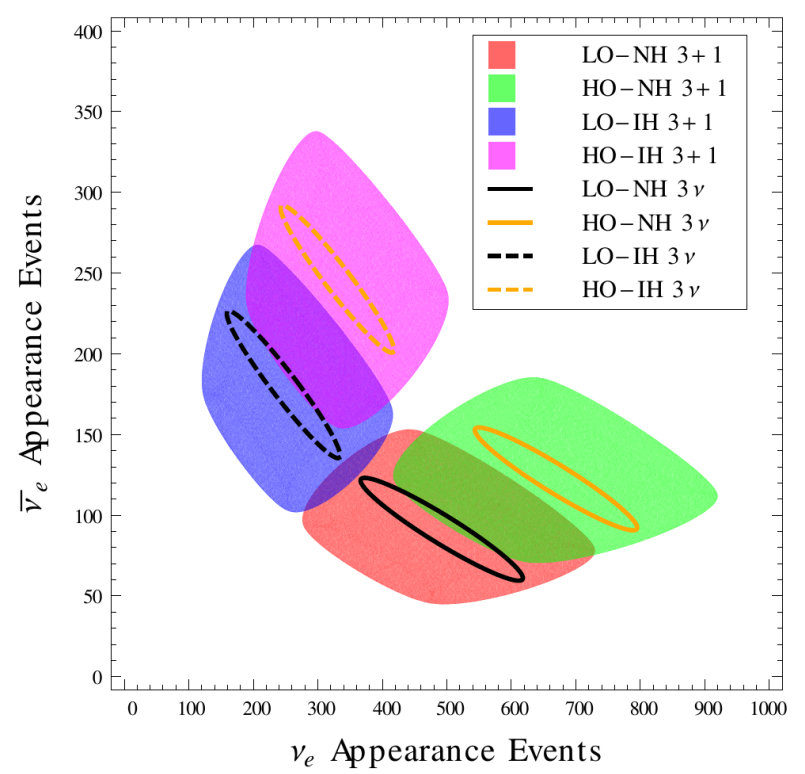

Figure 7. Bi-event plot for the DUNE setup. The ellipses display the 3-flavor scenario, while the colored blobs correspond to the $3+1$ framework. We take $\sin ^{2} \theta_{23}=0.42(0.58)$ as a representative value for the $\mathrm{LO}(\mathrm{HO})$. In the 3 -flavor ellipses, the running parameter is $\delta_{13}$ in the range $[-\pi, \pi]$. In the $3+1$ blobs, there are two running CP phases, $\delta_{13}$ and $\delta_{14}$. In the 4 -flavor case, we have assumed $\theta_{14}=\theta_{24}=9^{\circ}$ and $\theta_{34}=0^{\circ}$. Figure taken from [46].

6 As shown in [102,103], in 4 flavors, the condition $\theta_{23} \simeq 45^{\circ}$ implies an approximate realization of $\mu \leftrightarrow \tau$ symmetry, similar to what occurs in the standard 3-flavor scenario. Therefore, discovering that $\theta_{23}$ is maximal (non-maximal) would imply that $\mu \leftrightarrow \tau$ symmetry is unbroken (broken), independently of the existence of a light sterile neutrino. 
Figure 8 shows the DUNE discovery potential for nailing down the true octant as a function of true $\delta_{13}$. The left (right) panel refers to the true choice LO-NH (HO-NH). In both panels, we show also the results for the 3-flavor case (black curve). Regarding the $3+1$ scheme, we show the curves for four benchmark values of true $\delta_{14}\left(0^{\circ}, 180^{\circ},-90^{\circ}, 90^{\circ}\right)$. In the $3 v$ framework, the marginalization is performed over $\left(\theta_{23}, \delta_{13}\right)$ (test). In the $3+1$ scenario, we fix $\theta_{14}=\theta_{24}=9^{\circ}$ and $\theta_{34}=0$, and marginalize over $\left(\theta_{23}, \delta_{13}, \delta_{14}\right)$ (test). In all cases, we marginalize over the NMH. Figure 8 clearly depicts that in the $3+1$ scheme there exist unlucky combinations of $\delta_{13}$ (true) and $\delta_{14}$ (true) for which the octant sensitivity drops below the $2 \sigma$ level. We have also verified that for such combinations the energy spectra corresponding to the two octants are almost identical for neutrinos and antineutrinos. Therefore, even a broad-band experiment such as DUNE is not able to resolve the degeneracy introduced by a light sterile neutrino. Very recently, in [48], it has been shown that the situation may improve in T2HKK. However, a direct comparison with our results is not possible because in [48] the benchmark values assumed for the true mixing angles $\theta_{14}, \theta_{24}$, and $\theta_{34}$ are different (sensibly smaller) than those used in the present review.
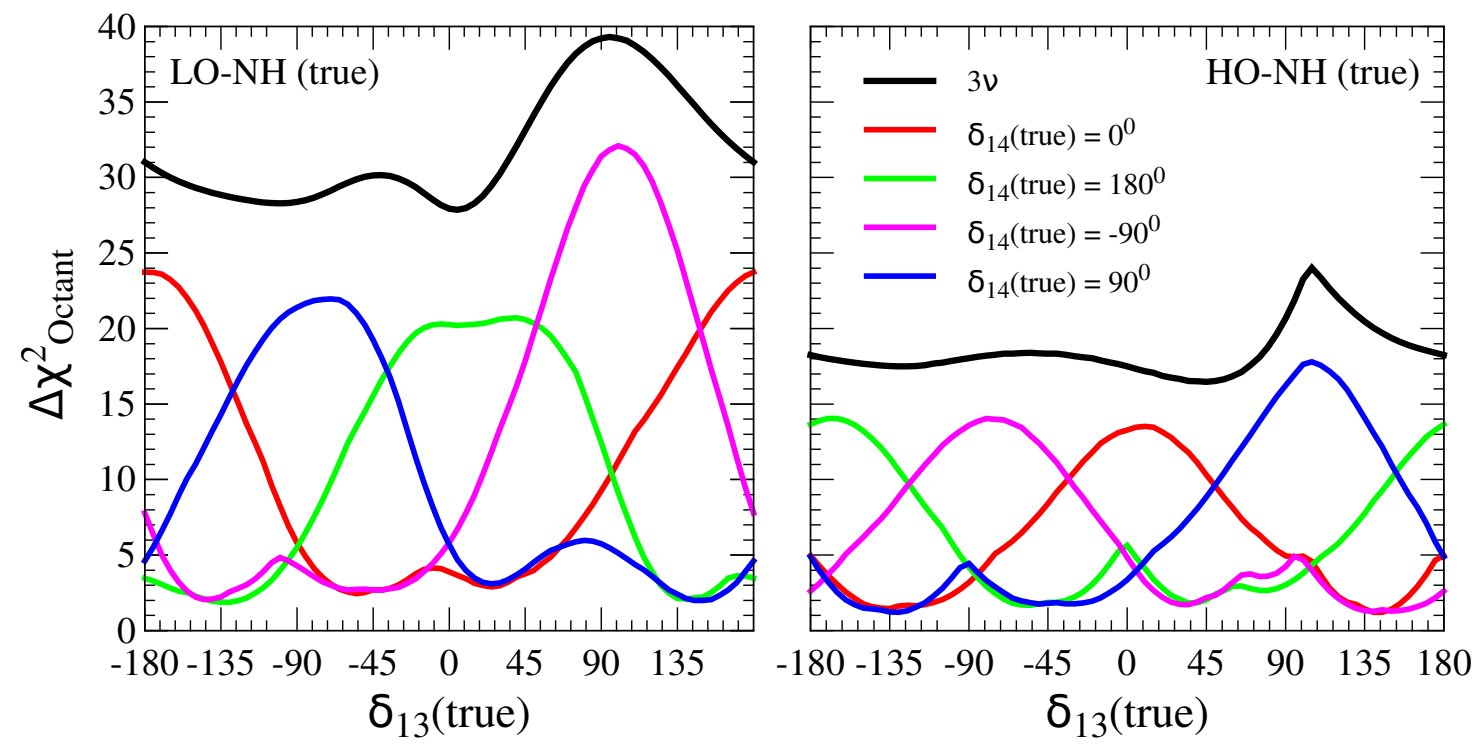

Figure 8. Sensitivity to the octant of $\theta_{23}$ as a function of true $\delta_{13}$ for LO-NH (left) and HO-NH (right) as the true option. We take $\sin ^{2} \theta_{23}=0.42(0.58)$ as representative value for $\mathrm{LO}(\mathrm{HO})$. In each panel, we present the results for the 3-flavor scenario (black line), and for the $3+1$ case we consider four different values of true $\delta_{14}$ (colored lines). Figure taken from [46].

\section{Conclusions}

Several anomalies observed in short-baseline neutrino experiments suggest the existence of new light sterile neutrino species. In this review, we discuss the potential role of long-baseline experiments in the searches of sterile neutrino properties and, in particular, the new CP-violation phases that appear in the enlarged $3+1$ scheme. We also assess the impact of light sterile states on the discovery potential of long-baseline experiments of important targets such as the standard 3-flavor CP violation, the neutrino mass hierarchy, and the octant of $\theta_{23}$. In the case of the discovery of sterile neutrinos, the planned LBL experiments will have a huge potential to explore the sterile neutrino properties and they will be complementary to SBL experiments.

Funding: We acknowledge partial support by the research grant number 2017W4HA7S “NAT-NET: Neutrino and Astroparticle Theory Network" under the program PRIN 2017 funded by the Italian Ministero dell'Istruzione, dell'Università e della Ricerca (MIUR) and by the research project TAsP funded by the Instituto Nazionale di Fisica Nucleare (INFN). 
Acknowledgments: We are grateful to three anonymous reviewers for the excellent feedback we received.

Conflicts of Interest: The author declares no conflict of interest.

\section{Appendix A. Conversion Probability in Matter}

In matter, the Hamiltonian can be expressed, in the flavor basis, as follows

$$
H=U K U^{\dagger}+V,
$$

with $K$ denoting the diagonal matrix of the wave numbers

$$
K=\operatorname{diag}\left(0, k_{21}, k_{31}, k_{41}\right),
$$

where $k_{i 1}=\Delta m_{i 1}^{2} / 2 E(i=2,3,4)$ and $V$ is the matrix describing the matter potential

$$
V=\operatorname{diag}\left(V_{C C}, 0,0,-V_{N C}\right),
$$

where

$$
V_{C C}=\sqrt{2} G_{F} N_{e}
$$

is the charged-current interaction potential of the electron neutrinos in a background of electrons having number density $N_{e}$, and

$$
V_{N C}=-\frac{1}{2} \sqrt{2} G_{F} N_{n}
$$

is the neutral-current interaction potential (equal for all the active neutrino species), where the background neutrons have number density $N_{n}$. We also introduce the positive-definite ratio

$$
r=-\frac{V_{N C}}{V_{C C}}=\frac{1}{2} \frac{N_{n}}{N_{e}}
$$

which, in the Earth crust, is given by $r \simeq 0.5$. With the purpose of simplifying the treatment of matter effects, we introduce the new basis

$$
\bar{v}=\bar{U}^{\dagger} v,
$$

where

$$
\bar{U}=\tilde{R}_{34} R_{24} \tilde{R}_{14}
$$

is the part of the mixing matrix defined in Equation (1), which incorporates only the rotations involving the fourth mass eigenstate. Therefore, the mixing matrix $U$ can be split as follows

$$
U=\bar{U} U_{3 v}
$$

where $U_{3 v}$ is the $4 \times 4$ matrix containing the standard 3-flavor mixing matrix in the $(1,2,3)$ sub-block. In the new basis, the Hamiltonian has the expression

$$
\bar{H}=\bar{H}^{\mathrm{kin}}+\bar{H}^{\mathrm{dyn}}=U_{3 v} K U_{3 v}^{\dagger}+\bar{U}^{\dagger} V \bar{U},
$$

where the first term is the kinematic term describing the oscillations in vacuum and the second one is the nonstandard dynamical term. As shown in [41] (see the Appendix therein), since $\left|k_{41}\right|$ is much larger than one and much larger than $\left|k_{21}\right|$ and $\left|k_{31}\right|$, one can reduce the dynamics to an effective 3-flavor one. Indeed, from Equation (A10), one can see that the $(4,4)$ element of $\bar{H}$ is much larger than all the other entries and the fourth eigenvalue of $\bar{H}$ is much bigger than the other three ones. As a 
consequence, the state $\bar{v}_{s}$ evolves independent of the others. Extracting the submatrix with indices $(1,2,3)$ from $\bar{H}$, one arrives at the $3 \times 3$ Hamiltonian

$$
\bar{H}_{3 v}=\bar{H}_{3 v}^{\text {kin }}+\bar{H}_{3 v}^{\text {dyn }}
$$

governing the $\left(\bar{v}_{e}, \bar{v}_{\mu}, \bar{v}_{\tau}\right)$ system, whose dynamical part has the form [41]

$$
\bar{H}_{3 v}^{\text {dyn }}=V_{C C}\left[\begin{array}{ccc}
\left|\bar{U}_{e 1}\right|^{2}+r\left|\bar{U}_{s 1}\right|^{2} & r \bar{U}_{s 1}^{*} \bar{U}_{s 2} & r \bar{U}_{s 1}^{*} \bar{U}_{s 3} \\
+ & r\left|\tilde{U}_{s 2}\right|^{2} & r \bar{U}_{s 2}^{*} \bar{U}_{s 3} \\
+ & \dagger & r\left|\bar{U}_{s 3}\right|^{2}
\end{array}\right],
$$

where we indicate with + the complex conjugate of the element with the same two indices inverted. For the derivation of Equation (A12), we exploit the relations $\bar{U}_{e 2}=\bar{U}_{e 3}=\bar{U}_{\mu 3}=0$. Considering the explicit expressions of the entries of $\bar{U}$ and taking their first-order expansion in the mixing angles $\theta_{i 4}$ $(i=1,2,3)$, Equation (A12) becomes

$$
\bar{H}_{3 v}^{\mathrm{dyn}} \approx V_{\mathrm{CC}}\left[\begin{array}{ccc}
1-(1-r) s_{14}^{2} & r \tilde{s}_{14} s_{24} & r \tilde{s}_{14} \tilde{s}_{34}^{*} \\
\dagger & r s_{24}^{2} & r s_{24} \tilde{S}_{34}^{*} \\
+ & \dagger & r s_{34}^{2}
\end{array}\right] .
$$

Equation (A13) shows that, if the sterile neutrino angles are zero $\left(\theta_{14}=\theta_{24}=\theta_{34}=0\right)$, one recovers the (diagonal) standard 3-flavor MSW Hamiltonian. In general, in the $3+1$ scenario, the Hamiltonian in Equation (A13) implies both diagonal and off-diagonal corrections. We can observe that these perturbations are formally equivalent to non-standard neutrino interactions (NSI) ${ }^{7}$

$$
\bar{H}_{3 v}^{\mathrm{dyn}} \approx V_{\mathrm{CC}}\left[\begin{array}{ccc}
1+\varepsilon_{e e} & \varepsilon_{e \mu} & \varepsilon_{e \tau} \\
\dagger & \varepsilon_{\mu \mu} & \varepsilon_{\mu \tau} \\
\dagger & \dagger & \varepsilon_{\tau \tau}
\end{array}\right] .
$$

This formal analogy is useful to interpret the sensitivity to the new dynamical effects implied by $3+1$ scheme. We recall that, in the $v_{\mu} \rightarrow v_{e}$ channel, the NSI that play the most pronounced impact are $\varepsilon_{e \mu}$ and $\varepsilon_{e \tau}$, while in the $v_{\mu} \rightarrow v_{\mu}$ channel $\varepsilon_{\mu \tau}$ has the largest effect. If the three new mixing angles have the same size $s_{14}^{2}=s_{24}^{2}=s_{34}^{2}=0.025$, all the perturbations in Equation (A14) are very small $\left(\left|\varepsilon_{\alpha \beta}\right| \simeq 0.01\right)$ and they have an unobservable impact. In such a case, the dynamics is basically identical to that of the standard 3-flavor scenario. On the other hand, if one allows the third mixing angle to take bigger values $\left(\theta_{34} \sim 30^{\circ}\right)$, the entries of the third column of the Hamiltonian in Equation (A14) can be noticeably bigger $\left(\left|\varepsilon_{e \tau}\right| \simeq\left|\varepsilon_{\mu \tau}\right| \simeq 0.04\right.$ and $\left.\left|\varepsilon_{\tau \tau}\right| \simeq 0.13\right)$. In this last case, one may expect a sizeable effect of $\varepsilon_{\mathcal{}} \equiv r \tilde{s}_{14} \tilde{\tilde{S}}_{34}^{*}$ in the $v_{\mu} \rightarrow v_{e}$ probability, and of $\varepsilon_{\mu \tau} \equiv r s_{24} \tilde{s}_{34}^{*}$ in the $v_{\mu} \rightarrow v_{\mu}$ probability. As a result, one expects that both channels should be sensitive to the third CP-phase $\delta_{34}$ since $\tilde{s}_{34}^{*} \equiv s_{34} e^{i \delta_{34}}$. Therefore, differently from the vacuum case, in matter, the flavor transitions are sensitive to $\theta_{34}$ and the related CP-phase $\delta_{34}$. The dependence of both channels from these parameters represents a very peculiar feature, because, in lucky circumstances (i.e., a large value of $\theta_{34}$ ), the LBL setups are sensitive not only to the two CP-phases $\delta_{13}$ and $\delta_{14}$, but also to $\delta_{34}$. Hence, the searches performed at LBL experiments (DUNE in particular) may provide access to the entire CPV sector implied by the $3+1$ scheme.

7 We stress that this should be taken only as formal analogy. In fact, the real NSI are mediated by heavy particles. In contrast, in the case of sterile neutrinos, there is no heavy mediator and the NSI-like structure of the Hamiltonian is connected to the circumstance that we are working in the new basis introduced in Equation (A7), which is rotated with respect to the original flavor basis. We mention that a similar analogy has been noticed in the field of solar neutrino conversion in the presence of sterile states [36]. 


\section{References}

1. Fukuda, Y.; et al. [Super-Kamiokande Collaboration]. Evidence for oscillation of atmospheric neutrinos. Phys. Rev. Lett. 1998, 81, 1562-1567. [CrossRef]

2. Ahmad, Q.R.; et al. [SNO Collaboration]. Measurement of the rate of $v_{e}+d \rightarrow p+p+e^{-}$interactions produced by ${ }^{8} B$ solar neutrinos at the Sudbury Neutrino Observatory. Phys. Rev. Lett. 2001, 87, 071301. [CrossRef] [PubMed]

3. Ahmad, Q.R.; et al. [SNO Collaboration]. Measurement of day and night neutrino energy spectra at SNO and constraints on neutrino mixing parameters. Phys. Rev. Lett. 2002, 89, 011302. [CrossRef] [PubMed]

4. Ahmad, Q.R.; et al. [SNO Collaboration]. Direct evidence for neutrino flavor transformation from neutral current interactions in the Sudbury Neutrino Observatory. Phys. Rev. Lett. 2002, 89, 011301. [CrossRef] [PubMed]

5. Eguchi, K.; et al. [KamLAND Collaboration]. First results from KamLAND: Evidence for reactor anti-neutrino disappearance. Phys. Rev. Lett. 2003, 90, 021802. [CrossRef] [PubMed]

6. Abazajian, K.N.; Acero, M.A.; Agarwalla, S.K.; Aguilar-Arevalo, A.A.; Albright, C.H.; Antusch, S.; Arguelles, C.A.; Balantekin, A.B.; Barenboim, G.; Barger, V.; et al. Light Sterile Neutrinos: A White Paper. arXiv 2012, arXiv:1204.5379. Available online: https:/ / arxiv.org/abs/1204.5379 (accessed on 6 March 2020).

7. Palazzo, A. Phenomenology of light sterile neutrinos: A brief review. Mod. Phys. Lett. A 2013, 28, 1330004. [CrossRef]

8. Gariazzo, S.; Giunti, C.; Laveder, M.; Li, Y.F.; Zavanin, E.M. Light sterile neutrinos. J. Phys. G 2016, $43,033001$. [CrossRef]

9. Giunti, C. Light Sterile Neutrinos: Status and Perspectives. Nucl. Phys. B 2016, 908, 336-353. [CrossRef]

10. Giunti, C.; Lasserre, T. eV-scale Sterile Neutrinos. Ann. Rev. Nucl. Part. Sci. 2019, 69, 163-190. [CrossRef]

11. Böser, S.; Buck, C.; Giunti, C.; Lesgourgues, J.; Ludhova, L.; Mertens, S.; Schukraft, A.; Wurm, M. Status of Light Sterile Neutrino Searches. Prog. Part. Nucl. Phys. 2019, 111, 103736. [CrossRef]

12. Capozzi, F.; Giunti, C.; Laveder, M.; Palazzo, A. Joint short- and long-baseline constraints on light sterile neutrinos. Phys. Rev. D 2017, 95, 033006. [CrossRef]

13. Gariazzo, S.; Giunti, C.; Laveder, M.; Li, Y.F. Updated Global $3+1$ Analysis of Short-BaseLine Neutrino Oscillations. J. High Energy Phys. 2017, 2017, 135. [CrossRef]

14. Dentler, M.; Hernandez-Cabezudo, A.; Kopp, J.; Machado, P.A.N.; Maltoni, M.; Martinez-Soler, I.; Schwetz, T. Updated Global Analysis of Neutrino Oscillations in the Presence of eV-Scale Sterile Neutrinos. J. High Energy Phys. 2018, 2018, 10. [CrossRef]

15. Diaz, A.; Arguelles, C.A.; Collin, G.H.; Conrad, J.M.; Shaevitz, M.H. Where Are We With Light Sterile Neutrinos? arXiv 2019, arXiv:1906.00045. Available online: https:/ / arxiv.org/abs/1906.00045 (accessed on 6 March 2020).

16. Aguilar-Arevalo, A.; et al. [LSND Collaboration]. Evidence for neutrino oscillations from the observation of anti-neutrino(electron) appearance in a anti-neutrino(muon) beam. Phys. Rev. D 2001, 64, 112007. [CrossRef]

17. Aguilar-Arevalo, A.A.; et al. [MiniBooNE Collaboration]. Significant Excess of ElectronLike Events in the MiniBooNE Short-Baseline Neutrino Experiment. Phys. Rev. Lett. 2018, 121, 221801. [CrossRef]

18. Mention, G.; Fechner, M.; Lasserre, T.; Mueller, T.; Lhuillier, D.; Cribier, M.; Letourneau, A. The Reactor Antineutrino Anomaly. Phys. Rev. D 2011, 83, 073006. [CrossRef]

19. Hampel, W.; et al. [GALLEX Collaboration]. Final results of the Cr-51 neutrino source experiments in GALLEX. Phys. Lett. B 1998, 420, 114-126. [CrossRef]

20. Abdurashitov, J.N.; Gavrin, V.N.; Girin, S.V.; Gorbachev, V.V.; Gurkina, P.P.; Ibragimova, T.V.; Kalikhov, A.V.; Khairnasov, N.G.; Knodel, T.V.; Matveev, V.A. Measurement of the response of a Ga solar neutrino experiment to neutrinos from an Ar-37 source. Phys. Rev. C 2006, 73, 045805. [CrossRef]

21. Adamson, P.; et al. [MINOS Collaboration]. Search for Sterile Neutrinos Mixing with Muon Neutrinos in MINOS. Phys. Rev. Lett. 2016, 117, 151803. [CrossRef] [PubMed]

22. Adamson, P.; et al. [MINOS+ Collaboration]. Search for sterile neutrinos in MINOS and MINOS+ using a two-detector fit. Phys. Rev. Lett. 2019, 122, 091803. [CrossRef] [PubMed]

23. Adamson, P.; et al. [The NOvA Collaboration]. Search for active-sterile neutrino mixing using neutral-current interactions in NOvA. Phys. Rev. D 2017, 96, 072006. [CrossRef] 
24. Abe, K.; et al. [T2K Collaboration]. Search for light sterile neutrinos with the T2K far detector Super-Kamiokande at a baseline of $295 \mathrm{~km}$. Phys. Rev. D 2019, 99, 071103. [CrossRef]

25. An, F.P.; et al. [Daya Bay Collaboration]. Improved Search for a Light Sterile Neutrino with the Full Configuration of the Daya Bay Experiment. Phys. Rev. Lett. 2016, 117, 151802. [CrossRef] [PubMed]

26. Adamson, P.; et al. [Daya Bay Collaboration, MINOS Collaboration]. Limits on Active to Sterile Neutrino Oscillations from Disappearance Searches in the MINOS, Daya Bay, and Bugey-3 Experiments. Phys. Rev. Lett. 2016, 117, 151801. [Addendum: Phys. Rev. Lett. 2016, 117, 209901]. [CrossRef] [PubMed]

27. Danilov, M. Recent results of the DANSS experiment. In Proceedings of the 2019 European Physical Society Conference on High Energy Physics (EPS-HEP2019), Ghent, Belgium, 10-17 July 2019.

28. Ko, Y.J.; et al. [NEOS Collaboration]. Sterile Neutrino Search at the NEOS Experiment. Phys. Rev. Lett. 2017, 118, 121802. [CrossRef]

29. Serebrov, A.P.; Ivochkin, V.G.; Samoilov, R.M.; Fomin, A.K.; Polyushkin, A.O.; Zinoviev, V.G.; Neustroev, P.V.; Golovtsov, V.L.; Chernyj, A.V.; Zherebtsov, O.M.; et al. First Observation of the Oscillation Effect in the Neutrino-4 Experiment on the Search for the Sterile Neutrino. JETP Lett. 2019, 109, 209-218. [CrossRef]

30. Ashenfelter, J.; et al. [PROSPECT Collaboration]. First search for short-baseline neutrino oscillations at HFIR with PROSPECT. Phys. Rev. Lett. 2018, 121, 251802. [CrossRef]

31. Almazán Molina, H.; Bernard, L.; Blanchet, A.; Bonhomme, A.; Buck, C.; Amo Sánchez, P.; Atmani, I.E.; Haser, H.; Kandzia, F.; Kox, S.; et al. Improved Sterile Neutrino Constraints from the STEREO Experiment with 179 Days of Reactor-On Data. arXiv 2019, arXiv:1912.06582. Available online: https:/ / cel.archivesouvertes.fr/LPSC/hal-02423748v1 (accessed on 6 March 2020).

32. Abe, K.; et al. [Super-Kamiokande Collaboration]. Limits on sterile neutrino mixing using atmospheric neutrinos in Super-Kamiokande. Phys. Rev. D 2015, 91, 052019. [CrossRef]

33. Aartsen, M.G.; et al. [IceCube Collaboration]. Searches for Sterile Neutrinos with the IceCube Detector. Phys. Rev. Lett. 2016, 117, 071801. [CrossRef] [PubMed]

34. Aartsen, M.G.; et al. [IceCube Collaboration]. Search for sterile neutrino mixing using three years of IceCube DeepCore data. Phys. Rev. D 2017, 95, 112002. [CrossRef]

35. Albert, A.; et al. [The ANTARES collaboration]. Measuring the atmospheric neutrino oscillation parameters and constraining the $3+1$ neutrino model with ten years of ANTARES data. J. High Energy Phys. 2019, 2019, 113. [CrossRef]

36. Palazzo, A. Testing the very-short-baseline neutrino anomalies at the solar sector. Phys. Rev. D 2011, 83, 113013. [CrossRef]

37. Palazzo, A. An estimate of $\theta_{14}$ independent of the reactor antineutrino flux determinations. Phys. Rev. D 2012, 85, 077301. [CrossRef]

38. Giunti, C.; Li, Y.F. Matter Effects in Active-Sterile Solar Neutrino Oscillations. Phys. Rev. D 2009, 80, 113007. [CrossRef]

39. Lasserre, T. Light Sterile Neutrinos in Particle Physics: Experimental Status. Phys. Dark Univ. 2014, 4, 81-85. [CrossRef]

40. Nunokawa, H.; Peres, O.L.G.; Zukanovich Funchal, R. Probing the LSND mass scale and four neutrino scenarios with a neutrino telescope. Phys. Lett. B 2003, 562, 279-290. [CrossRef]

41. Klop, N.; Palazzo, A. Imprints of CP violation induced by sterile neutrinos in T2K data. Phys. Rev. D 2015, 91, 073017. [CrossRef]

42. Hollander, D.; Mocioiu, I. Minimal $3+2$ sterile neutrino model at LBNE. Phys. Rev. D 2015, 91, 013002. [CrossRef]

43. Berryman, J.M.; de Gouvêa, A.; Kelly, K.J.; Kobach, A. Sterile neutrino at the Deep Underground Neutrino Experiment. Phys. Rev. D 2015, 92, 073012. [CrossRef]

44. Gandhi, R.; Kayser, B.; Masud, M.; Prakash, S. The impact of sterile neutrinos on CP measurements at long baselines. J. High Energy Phys. 2015, 2015, 39. [CrossRef]

45. Agarwalla, S.K.; Chatterjee, S.S.; Palazzo, A. Physics Reach of DUNE with a Light Sterile Neutrino. J. High Energy Phys. 2016, 2016, 16, [CrossRef]

46. Agarwalla, S.K.; Chatterjee, S.S.; Palazzo, A. Octant of $\theta_{23}$ in danger with a light sterile neutrino. Phys. Rev. Lett. 2017, 118, 031804. [CrossRef] [PubMed]

47. Coloma, P.; Forero, D.V.; Parke, S.J. DUNE Sensitivities to the Mixing between Sterile and Tau Neutrinos. J. High Energy Phys. 2018, 2018, 79. [CrossRef] 
48. Choubey, S.; Dutta, D.; Pramanik, D. Imprints of a light Sterile Neutrino at DUNE, T2HK and T2HKK. Phys. Rev. D 2017, 96, 056026. [CrossRef]

49. Choubey, S.; Dutta, D.; Pramanik, D. Measuring the Sterile Neutrino CP Phase at DUNE and T2HK. Eur. Phys. J. A 2017, 78, 339. [CrossRef]

50. Agarwalla, S.K.; Chatterjee, S.S.; Palazzo, A. Signatures of a Light Sterile Neutrino in T2HK. J. High Energy Phys. 2018, 2018, 91. [CrossRef]

51. Haba, N.; Mimura, Y.; Yamada, T. On $\theta_{23}$ Octant Measurement in $3+1$ Neutrino Oscillations in T2HKK. arXiv 2018, arXiv:1812.10940. Available online: https:/ / arxiv.org/abs/1812.10940 (accessed on 6 March 2020).

52. Donini, A.; Meloni, D. The $2+2$ and $3+1$ four family neutrino mixing at the neutrino factory. Eur. Phys. J. C 2001, 22, 179-186. [CrossRef]

53. Donini, A.; Lusignoli, M.; Meloni, D. Telling three neutrinos from four neutrinos at the neutrino factory. Nucl. Phys. B 2002, 624, 405-422. [CrossRef]

54. Donini, A.; Maltoni, M.; Meloni, D.; Migliozzi, P.; Terranova, F. $3+1$ sterile neutrinos at the CNGS. J. High Energy Phys. 2007, 2007, 13. [CrossRef]

55. Dighe, A.; Ray, S. Signatures of heavy sterile neutrinos at long baseline experiments. Phys. Rev. D 2007, 76, 113001. [CrossRef]

56. Donini, A.; Fuki, K.I.; Lopez-Pavon, J.; Meloni, D.; Yasuda, O. The Discovery channel at the Neutrino Factory: Numu to nutau pointing to sterile neutrinos. J. High Energy Phys. 2009, 2009, 41. [CrossRef]

57. Yasuda, O. Sensitivity to sterile neutrino mixings and the discovery channel at a neutrino factory. Physics beyond the standard models of particles, cosmology and astrophysics. In Proceedings of the 5th International Conference, Beyond 2010, Cape Town, South Africa, 1-6 February 2010; pp. 300-313.

58. Meloni, D.; Tang, J.; Winter, W. Sterile neutrinos beyond LSND at the Neutrino Factory. Phys. Rev. D 2010, 82, 093008. [CrossRef]

59. Bhattacharya, B.; Thalapillil, A.M.; Wagner, C.E.M. Implications of sterile neutrinos for medium/long-baseline neutrino experiments and the determination of $\theta_{13}$. Phys. Rev. D 2012, 85, 073004. [CrossRef]

60. Donini, A.; Hernandez, P.; Lopez-Pavon, J.; Maltoni, M.; Schwetz, T. The minimal $3+2$ neutrino model versus oscillation anomalies. J. High Energy Phys. 2012, 2012, 161. [CrossRef]

61. Gandhi, R.; Kayser, B.; Prakash, S.; Roy, S. What measurements of neutrino neutral current events can reveal. J. High Energy Phys. 2017, 2017, 202. [CrossRef]

62. Kim, C.S.; López Castro, G.; Sahoo, D. Constraints on a sub-eV scale sterile neutrino from nonoscillation measurements. Phys. Rev. D 2018, 98, 115021. [CrossRef]

63. Giunti, C.; Li, Y.F.; Zhang, Y.Y. KATRIN bound on $3+1$ active-sterile neutrino mixing and the reactor antineutrino anomaly. arXiv 2019, arXiv:1912.12956. Available online: https:/ / arxiv.org/abs/1912.12956 (accessed on 6 March 2020).

64. Goswami, S.; Rodejohann, W. Constraining mass spectra with sterile neutrinos from neutrinoless double beta decay, tritium beta decay and cosmology. Phys. Rev. D 2006, 73, 113003. [CrossRef]

65. Girardi, I.; Meroni, A.; Petcov, S.T. Neutrinoless Double Beta Decay in the Presence of Light Sterile Neutrinos. J. High Energy Phys. 2013, 2013, 146. [CrossRef]

66. De Salas, P.F.; Forero, D.V.; Ternes, C.A.; Tortola, M.; Valle, J.W.F. Status of neutrino oscillations 2018: $3 \sigma$ hint for normal mass ordering and improved CP sensitivity. Phys. Lett. B 2018, 782, 633-640. [CrossRef]

67. Capozzi, F.; Lisi, E.; Marrone, A.; Palazzo, A. Current unknowns in the three neutrino framework. Prog. Part. Nucl. Phys. 2018, 102, 48-72. [CrossRef]

68. Esteban, I.; Gonzalez-Garcia, M.C.; Hernandez-Cabezudo, A.; Maltoni, M.; Schwetz, T. Global analysis of three-flavour neutrino oscillations: Synergies and tensions in the determination of $\theta_{2} 3, \delta_{C} P$, and the mass ordering. J. High Energy Phys. 2019, 2019, 106. [CrossRef]

69. Acciarri, R.; et al. [DUNE Collaboration]. Long-Baseline Neutrino Facility (LBNF) and Deep Underground Neutrino Experiment (DUNE) Conceptual Design Report Volume 1: The LBNF and DUNE Projects. arXiv 2016, arXiv:1601.054712016. Available online: https://cds.cern.ch/record/2126238 (accessed on 6 March 2020).

70. Acciarri, R.; et al. [DUNE collaboration]. Long-Baseline Neutrino Facility (LBNF) and Deep Underground Neutrino Experiment (DUNE). arXiv 2015, arXiv:1512.06148. Available online: http:/ /xxx.lanl.gov/abs/ 1512.06148 (accessed on 6 March 2020). 
71. Strait, J.; McCluskey, E.; Lundin, T.; Willhite, J.; Hamernik, T.; Papadimitriou, V.; Marchionni, A.; Kim, M.J.; Nessi, M.; Montanari, D.; et al. Long-Baseline Neutrino Facility (LBNF) and Deep Underground Neutrino Experiment (DUNE) Conceptual Design Report Volume 3: Long-Baseline Neutrino Facility for DUNE June 24, 2015. arXiv 2016, arXiv:1601.05823. Available online: https://arxiv.org/abs/1601.05823 (accessed on 6 March 2020).

72. Acciarri, R.; et al. [DUNE Collaboration]. Long-Baseline Neutrino Facility (LBNF) and Deep Underground Neutrino Experiment (DUNE) Conceptual Design Report, Volume 4 The DUNE Detectors at LBNF. arXiv 2016, arXiv:1601.02984. Available online: https:/ /arxiv.org/abs/1601.02984 (accessed on 6 March 2020).

73. Adams, C.; et al. [LBNE Collaboration]. Scientific Opportunities with the Long-Baseline Neutrino Experiment; Fermi National Accelerator Lab. (FNAL): Batavia, IL, USA, 2013.

74. Agarwalla, S.K.; Li, T.; Rubbia, A. An Incremental approach to unravel the neutrino mass hierarchy and CP violation with a long-baseline Superbeam for large $\theta_{13}$. J. High Energy Phys. 2012, 2012, 154. [CrossRef]

75. Bishai, M. (Brookhaven National Laboratory, Upton, NY 11973, USA). Personal communication, 2012.

76. Abe, K.; Abe, T.; Aihara, H.; Fukuda, Y.; Hayato, Y.; Huang, K.; Ichikawa, A.K.; Ikeda, M.; Inoue, K.; Ishino, H.; et al. Letter of Intent: The Hyper-Kamiokande Experiment-Detector Design and Physics Potential. arXiv 2011, arXiv:1109.3262. Available online: https:/ / arxiv.org/abs/1109.3262 (accessed on 6 March 2020).

77. Abe, K.; et al. [Hyper-Kamiokande Working Group]. A Long Baseline Neutrino Oscillation Experiment Using J-PARC Neutrino Beam and Hyper-Kamiokande. arXiv 2014, arXiv:1412.4673. Available online: https: / / arxiv.org/abs/1412.4673 (accessed on 6 March 2020).

78. Abe, K.; et al. [Hyper-Kamiokande Proto-Collaboration]. Physics potential of a long-baseline neutrino oscillation experiment using a J-PARC neutrino beam and Hyper-Kamiokande. Prog. Theor. Exp. Phys. 2015, 2015, 053 C02.

79. Para, A.; Szleper, M. Neutrino oscillations experiments using off-axis NuMI beam. arXiv 2001, arXiv:hep-ex/0110032. Available online: https:/ / arxiv.org/abs/hep-ex/0110032 (accessed on 6 March 2020).

80. Abe, K.; et al. [Hyper-Kamiokande proto-Collaboration]. Physics potentials with the second Hyper-Kamiokande detector in Korea. Prog. Theor. Exp. Phys. 2018, 2018, 063 C01.

81. Baussan, E.; Blennow, B.; Bogomilov, M.; Bouquerel, E.; Caretta, O.; Cederkäll, J.; Christiansen, P.; Coloma, P.; Cupial, P.; Danared, H.; et al. A very intense neutrino super beam experiment for leptonic CP violation discovery based on the European spallation source linac. Nucl. Phys. B 2014, 885, 127-149. [CrossRef]

82. Dracos, M. The ESS $v$ SB Project for Leptonic CP Violation Discovery based on the European Spallation Source Linac. Nucl. Part. Phys. Proc. 2016, 273, 1726-1731. [CrossRef]

83. Dracos, M. The European Spallation Source neutrino Super Beam. In Proceedings of the Prospects in Neutrino Physics (NuPhys2017), London, UK, 20-22 December 2017; pp. 33-41.

84. Dracos, M.; Ekelof, T. Neutrino CP Violation with the ESS neutrino Super Beam (ESS vSB). PoS 2019, $12,524$.

85. Martinez, E.F. (IFT, Madrid, Spain). Personal communication, 2013.

86. Agostino, L.; Buizza-Avanzini, M.; Dracos, M.; Duchesneau, D.; Marafini, M.; Mezzetto, M.; Mosca, L.; Patzak, T.; Tonazzo, A.; Vassilopoulos, N. Study of the performance of a large scale water-Cherenkov detector (MEMPHYS). J. Cosmol. Astropart. Phys. 2013, 2013, 24. [CrossRef]

87. Agostino, L. (LPNHE Paris, Université Pierre et Marie Curie, Université Paris Diderot, CNRS/IN2P3, Paris 75252, France). Personal communication, 2013.

88. Agarwalla, S.K.; Choubey, S.; Prakash, S. Probing Neutrino Oscillation Parameters using High Power Superbeam from ESS. J. High Energy Phys. 2014, 2014, 20. [CrossRef]

89. Kumar Agarwalla, S.; Chatterjee, S.S.; Palazzo, A. Physics Potential of ESS $v$ SB in the presence of a Light Sterile Neutrino. J. High Energy Phys. 2019, 2019, 174. [CrossRef]

90. Huber, P.; Lindner, M.; Winter, W. Simulation of long-baseline neutrino oscillation experiments with GLoBES (General Long Baseline Experiment Simulator). Comput. Phys. Commun. 2005, 167, 195. [CrossRef]

91. Huber, P.; Kopp, J.; Lindner, M.; Rolinec, M.; Winter, W. New features in the simulation of neutrino oscillation experiments with GLoBES 3.0: General Long Baseline Experiment Simulator. Comput. Phys. Commun. 2007, 177, 432-438. [CrossRef]

92. Dziewonski, A.M.; Anderson, D.L. Preliminary Reference Earth Model. Phys. Earth Planet. Inter. 1981, 25, 297-356. [CrossRef] 
93. Huber, P.; Lindner, M.; Winter, W. Superbeams versus neutrino factories. Nucl. Phys. B 2002, 645, 3-48. [CrossRef]

94. Fogli, G.L.; Lisi, E.; Marrone, A.; Montanino, D.; Palazzo, A. Getting the most from the statistical analysis of solar neutrino oscillations. Phys. Rev. D 2002, 66, 053010. [CrossRef]

95. Blennow, M.; Coloma, P.; Huber, P.; Schwetz, T. Quantifying the sensitivity of oscillation experiments to the neutrino mass ordering. J. High Energy Phys. 2014, 2014, 28. [CrossRef]

96. Agarwalla, S.K.; Chatterjee, S.S.; Dasgupta, A.; Palazzo, A. Discovery Potential of T2K and NOvA in the Presence of a Light Sterile Neutrino. J. High Energy Phys. 2016, 2016, 111. [CrossRef]

97. Mohapatra, R.; Smirnov, A. Neutrino Mass and New Physics. Ann. Rev. Nucl. Part. Sci. 2006, 56, 569-628. [CrossRef]

98. Albright, C.H.; Chen, M.C. Model Predictions for Neutrino Oscillation Parameters. Phys. Rev. D 2006, 74, 113006. [CrossRef]

99. Altarelli, G.; Feruglio, F. Discrete Flavor Symmetries and Models of Neutrino Mixing. Rev. Mod. Phys. 2010, 82, 2701-2729. [CrossRef]

100. King, S.F.; Merle, A.; Morisi, S.; Shimizu, Y.; Tanimoto, M. Neutrino Mass and Mixing: From Theory to Experiment. New J. Phys. 2014, 16, 045018. [CrossRef]

101. King, S.F. Models of Neutrino Mass, Mixing and CP Violation. J. Phys. G 2015, 42, 123001. [CrossRef]

102. Merle, A.; Morisi, S.; Winter, W. Common origin of reactor and sterile neutrino mixing. J. High Energy Phys. 2014, 2014, 39. [CrossRef]

103. Rivera-Agudelo, D.C.; Pérez-Lorenzana, A. Generating $\theta_{13}$ from sterile neutrinos in $\mu-\tau$ symmetric models. Phys. Rev. D 2015, 92, 073009. [CrossRef]

104. Fukuyama, T.; Nishiura, H. Mass matrix of Majorana neutrinos. arXiv 1997, arXiv:hep-ph/9702253v1. Available online: https:/ /arxiv.org/abs/hep-ph/9702253v1 (accessed on 6 March 2020).

105. Mohapatra, R.N.; Nussinov, S. Bimaximal neutrino mixing and neutrino mass matrix. Phys. Rev. D 1999, 60, 013002. [CrossRef]

106. Lam, C. A 2-3 symmetry in neutrino oscillations. Phys. Lett. B 2001, 507, 214-218. [CrossRef]

107. Harrison, P.; Scott, W. mu-tau reflection symmetry in lepton mixing and neutrino oscillations. Phys. Lett. $B$ 2002, 547, 219-228. [CrossRef]

108. Kitabayashi, T.; Yasue, M. S(2L) permutation symmetry for left-handed mu and tau families and neutrino oscillations in an SU(3)-L $\times$ SU(1)-N gauge model. Phys. Rev. D 2003, 67, 015006. [CrossRef]

109. Grimus, W.; Lavoura, L. A Discrete symmetry group for maximal atmospheric neutrino mixing. Phys. Lett. $B$ 2003, 572, 189-195. [CrossRef]

110. Koide, Y. Universal texture of quark and lepton mass matrices with an extended flavor 2->3 symmetry. Phys. Rev. D 2004, 69, 093001. [CrossRef]

111. Mohapatra, R.; Rodejohann, W. Broken mu-tau symmetry and leptonic CP violation. Phys. Rev. D 2005, 72, 053001. [CrossRef]

112. Ma, E. Plato's fire and the neutrino mass matrix. Mod. Phys. Lett. A 2002, 17, 2361-2370. [CrossRef]

113. Ma, E.; Rajasekaran, G. Softly broken A(4) symmetry for nearly degenerate neutrino masses. Phys. Rev. D 2001, 64, 113012. [CrossRef]

114. Babu, K.; Ma, E.; Valle, J. Underlying A(4) symmetry for the neutrino mass matrix and the quark mixing matrix. Phys. Lett. B 2003, 552, 207-213. [CrossRef]

115. Grimus, W.; Lavoura, L. S(3) $\times$ Z(2) model for neutrino mass matrices. J. High Energy Phys. 2005, $2005,13$. [CrossRef]

116. Ma, E. Tetrahedral family symmetry and the neutrino mixing matrix. Mod. Phys. Lett. A 2005, 20, 2601-2606. [CrossRef]

117. Raidal, M. Relation between the neutrino and quark mixing angles and grand unification. Phys. Rev. Lett. 2004, 93, 161801. [CrossRef]

118. Minakata, H.; Smirnov, A.Y. Neutrino mixing and quark-lepton complementarity. Phys. Rev. D 2004, 70, 073009. [CrossRef]

119. Ferrandis, J.; Pakvasa, S. Quark-lepton complenmentarity relation and neutrino mass hierarchy. Phys. Rev. D 2005, 71, 033004. [CrossRef]

120. Antusch, S.; King, S.F.; Mohapatra, R.N. Quark-lepton complementarity in unified theories. Phys. Lett. 2005, B618, 150-161. [CrossRef] 
121. Hall, L.J.; Murayama, H.; Weiner, N. Neutrino mass anarchy. Phys. Rev. Lett. 2000, 84, 2572-2575. [CrossRef]

122. De Gouvea, A.; Murayama, H. Neutrino Mixing Anarchy: Alive and Kicking. Phys. Lett. 2015, B747, 479-483. [CrossRef]

123. Fogli, G.L.; Lisi, E. Tests of three-flavor mixing in long-baseline neutrino oscillation experiments. Phys. Rev. D 1996, 54, 3667-3670. [CrossRef]

124. Barger, V.; Marfatia, D.; Whisnant, K. Breaking eight-fold degeneracies in neutrino CP violation, mixing, and mass hierarchy. Phys. Rev. D 2002, 65, 073023. [CrossRef]

125. Minakata, H.; Nunokawa, H. Exploring neutrino mixing with low energy superbeams. J. High Energy Phys. 2001, 2001, 1. [CrossRef]

126. Hiraide, K.; Minakata, H.; Nakaya, T.; Nunokawa, H.; Sugiyama, H.; Teves, W.J.C.; Zukanovich Funchal, R. Resolving $\theta_{23}$ degeneracy by accelerator and reactor neutrino oscillation experiments. Phys. Rev. D 2006, 73, 093008. [CrossRef]

127. Burguet-Castell, J.; Gavela, M.; Gomez-Cadenas, J.; Hernandez, P.; Mena, O. Superbeams plus neutrino factory: The Golden path to leptonic CP violation. Nucl. Phys. B 2002, 646, 301-320. [CrossRef]

128. Agarwalla, S.K.; Prakash, S.; Sankar, S.U. Resolving the octant of theta23 with T2K and NOvA. J. High Energy Phys. 2013, 2013, 131. [CrossRef]

129. Machado, P.A.N.; Minakata, H.; Nunokawa, H.; Zukanovich Funchal, R. What can we learn about the lepton CP phase in the next 10 years? J. High Energy Phys. 2014, 2014, 109. [CrossRef]

130. Minakata, H.; Parke, S.J. Correlated, precision measurements of $\theta_{23}$ and $\delta$ using only the electron neutrino appearance experiments. Phys. Rev. D 2013, 87, 113005. [CrossRef]

131. Chatterjee, A.; Ghoshal, P.; Goswami, S.; Raut, S.K. Octant sensitivity for large theta(13) in atmospheric and long baseline neutrino experiments. J. High Energy Phys. 2013, 2013, 10. [CrossRef]

132. Agarwalla, S.K.; Prakash, S.; Uma Sankar, S. Exploring the three flavor effects with future superbeams using liquid argon detectors. J. High Energy Phys. 2014, 2014, 87. [CrossRef]

133. Bass, M.; Bishai, M.; Cherdack, D.; Diwan, M.; Djurcic, Z.; Hernandez, J.; Lundberg, B.; Paolone, V.; Qian, X.; Rameika, R.; et al. Baseline optimization for the measurement of $\mathrm{CP}$ violation, mass hierarchy, and $\theta_{23}$ octant in a long-baseline neutrino oscillation experiment. Phys. Rev. D 2015, 91, 052015. [CrossRef]

134. Bora, K.; Dutta, D.; Ghoshal, P. Determining the octant of $\theta_{23}$ at LBNE in conjunction with reactor experiments. Mod. Phys. Lett. A 2015, 30, 1550066. [CrossRef]

135. Das, C.R.; Maalampi, J.; Pulido, J.; Vihonen, S. Determination of the $\theta_{23}$ octant in LBNO. J. High Energy Phys. 2015, 2015, 48. [CrossRef]

136. Nath, N.; Ghosh, M.; Goswami, S. The physics of antineutrinos in DUNE and determination of octant and $\delta_{C P}$. Nucl. Phys. B 2016, 913, 381-404. [CrossRef]

(C) 2020 by the authors. Licensee MDPI, Basel, Switzerland. This article is an open access article distributed under the terms and conditions of the Creative Commons Attribution (CC BY) license (http://creativecommons.org/licenses/by/4.0/). 